\title{
Optimal capital injection and dividend distribution for growth restricted diffusion models with bankruptcy
}

\author{
Jinxia Zhu ${ }^{a}$ and Hailiang Yang $^{b}$ \\ ${ }^{a}$ School of Risk and Actuarial Studies, The University of New South Wales, Kensington Campus, NSW \\ 2052, Australia; E-mail: jinxia.zhu@unsw.edu.au \\ ${ }^{b}$ Department of Statistics and Actuarial Science, The University of Hong Kong, Pokfulam Road, Hong \\ Kong; E-mail: hlyang@hku.hk
}

\begin{abstract}
We consider the optimal capital injection and dividend control problem for a company with the possibility of bankruptcy. The surplus process of the company is modeled by a growth restricted diffusion model with drift and diffusion coefficients being functions of the surplus process. The company can control the dividend payments and capital injections with the goal of maximizing the expectation of the total discounted dividends minus the total cost of capital injections up to the time of bankruptcy. We distinguish three cases and provide optimality results for each case.
\end{abstract}

JEL Classification C61; C02

Keywords: Capital injection, Control, Diffusion, Dividend.

\section{Introduction}

The optimal dividend control problem has attracted significant interest in the literature; see Albrecher and Thonhauser (2009), Avanzi (2009), Schmidli (2008) and the references therein. Many works model the underlying surplus process by a Brownian motion with drift (see for example, Asmussen et al. (2000), Guo et al. (2004), Yang et al. (2005), Cadenillas et al. (2006) and He and Liang (2009)). The dividend optimization problem for more general diffusions are studied in Shreve et al. (1984), Højgaard and Taksar (2001), Bäuerle (2004) and Alvarez and Virtanen (2006), Cadenillas et al. (2007), Paulsen (2008), Zhu (2015) and references therein.

The dividend optimization problem with the inclusion of capital injections which aims at maximizing the expected total discounted dividend payments minus the expected total discounted costs of capital injections is studied in Shreve et al. (1984) and has gained much interest in the recent literature. Shreve et al. (1984) investigated this optimization problem (framed as a reflection problem in the paper) for a general diffusion model subject to the constraint that the surplus process remains non-negative all the times (guaranteed via

\footnotetext{
${ }^{0}$ Address correspondence to Jinxia Zhu, School of Risk and Actuarial Studies, The University of New South Wales, Kensington Campus, NSW 2052, Australia; E-mail: jinxia.zhu@unsw.edu.au.
} 
capital injections whenever necessary even though this may not be optimal in some situations). Løkka and Zervos (2008), however, addressed the optimal dividend and issuance of equity policies control problem with the possibility of bankruptcy for a Brownian motion model. He and Liang (2008) studied a similar problem with the addition of proportional reinsurance policy for the Brownian motion model. Meng and Siu (2011) applied the viscosity solution approach to study the optimal capital injection and dividend control problem for the Brownian motion model where there are fixed and proportional costs for each dividend payment. Sethi and Taksar (2002) addressed the optimal dividend and financing control problem for a more general diffusion model. However, the paper does not taking into consideration of the possibility of bankruptcy (which generally occurs when the surplus drops below a certain level, say 0) at all.

This paper studies the optimal capital injection and dividend control for a class of growth restricted diffusion models with the possibility of bankruptcy. As in Løkka and Zervos (2008), we assume that the objective is to maximize the expected discounted dividend payments minus the expected discounted costs of capital injections up to the time of ruin, which is defined to be the moment that the surplus process drops below 0 for the first time. Our work can be considered as a generalization of the control problem in Løkka and Zervos (2008) in that both the drift and diffusion coefficients of the diffusion model in our paper are functions of the level of the surplus and therefore the model in our paper includes the Brownian motion model considered in Løkka and Zervos (2008) as a special case. The major technical difficulty in our extended model is caused by the fact that the ordinary differential equation (ODE) involved in the associated HamiltonJacobi-Bellmen (HJB) equation, unlike the constant coefficient ODE in Løkka and Zervos (2008), has varying coefficients that are general functions (with unspecified forms) of the variable. This means that we will not be able to derive the explicit form of the solution, let alone to obtain a simple exponential form that the solution in Løkka and Zervos (2008) has. The explicit and especially exponential form in Løkka and Zervos (2008) allows the authors to derive analytical properties directly, which plays a crucial role in finding the final optimal results.

We organize the rest of the paper as follows. In Section 2, we provide the formulation of the optimization problem. In Section 3, we study the functions that are solutions to the ordinary differential equation involved in the associated HJB equation and some functions constructed from these solutions. We distinguish and analyze 3 cases, and present the optimality results for each case in Section 4. We illustrate the results with two examples in Section 5. Concluding remarks are provided in Section 6.

\section{Problem Formulation}

Consider a probability space $(\Omega, \mathcal{F}, \mathrm{P})$. Let $\left\{W_{t} ; t \geq 0\right\}$ be a standard Brownian motion and $\left\{\mathcal{F}_{t} ; t \geq 0\right\}$ be the minimal complete $\sigma$-field generated by the stochastic process $\left\{W_{t} ; t \geq 0\right\}$. Let $X_{t}$ denote the cash flow surplus at time $t$ of a company in 
absence of capital injections and dividend payments. Assume that the initial value of the surplus process, $X_{0-}$, is $\mathcal{F}_{0}$ measurable, and that $X_{t}$ has the following dynamics

$$
\mathrm{d} X_{t}=\mu\left(X_{t-}\right) \mathrm{d} t+\sigma\left(X_{t-}\right) \mathrm{d} W_{t}, t \geq 0
$$

where the functions $\mu(\cdot)$ and $\sigma(\cdot)$ are Lipschitz continuous, differentiable and grow at most linearly on $[0, \infty)$. Let $\delta$ denote the force of interest for the valuation of shareholders' cash flows. Furthermore, we assume that the function $\sigma(\cdot)$ is positive and non-vanishing, and $\mu^{\prime}(x)<\delta$ for $x \geq 0$.

Remark 2.1. The diffusion mode under the constraint, $\mu^{\prime}(x)<\delta$ for $x \geq 0$, is general compared with most of the models used in the literature of the dividend optimization problem with or without the inclusion of capital injection control. In the literature, most of the works used the drifted Brownian motion model (i.e., $\mu(\cdot)=$ a constant and $\sigma(\cdot)=$ a constant), a couple of papers considered the Brownian model compounded by a constant force of interest (i.e. $\mu(x)=p+r x$ with $p \geq 0$ and $r<\delta, \sigma(x)=a$ constant) and one paper investigated the mean-reverting process (i.e. $\mu(\cdot)=c-r x$; see Cadenillas et al. (2007)). All these are all special cases of the growth restricted diffusions considered in this paper.

The company can distribute part of its assets to the shareholders as dividends and the shareholders can reinvest (under no obligation) via capital injections. There are transaction costs associated with dividend payments and capital injections. For each dollar of reinvestment, it includes $c(c>0)$ dollars of transaction cost and hence leads to an increase of $1-c$ dollars in the surplus through capital injections. Let $C_{t}$ denote the cumulative amount of capital injections up to time $t$. Then the total cost for capital injections up to time $t$ is $\frac{C_{t}}{1-c}$. For each dollar of dividends received by the shareholders, there will be $d(d>0)$ dollars of transaction cost. Let $D_{t}$ denote the cumulative amount of dividends paid out by the company up to time $t$. Then the total amount of dividends received by the shareholders up to time $t$ is $\frac{D_{t}}{1+d}$. Both $\left\{C_{t} ; t \geq 0\right\}$ and $\left\{D_{t} ; t \geq 0\right\}$ are controllable by the company. We call $\pi:=\left\{\left(C_{t}, D_{t}\right) ; t \geq 0\right\}$ a control strategy.

The dynamics of the controlled surplus process (by the strategy $\pi$ ) is

$$
\mathrm{d} X_{t}^{\pi}=\mu\left(X_{t-}^{\pi}\right) \mathrm{d} t+\sigma\left(X_{t-}^{\pi}\right) \mathrm{d} W_{t}-\mathrm{d} D_{t}+\mathrm{d} C_{t}, t \geq 0 .
$$

Definition 2.1. A strategy $\pi=\left\{\left(C_{t}, D_{t}\right) ; t \geq 0\right\}$ is said to be admissible if (i) both $\left\{C_{t} ; t \geq 0\right\}$ and $\left\{D_{t} ; t \geq 0\right\}$ are nonnegative, increasing, càdlàg, and $\left\{\mathcal{F}_{t} ; t \geq 0\right\}$-adapted processes, (ii) $C_{0-}=D_{0-}=0$, and (iii) $\Delta D_{t} \leq X_{t}^{\pi}$.

We use $\Pi$ to denote the class of admissible strategies.

Define the time to bankruptcy by

$$
T^{\pi}=\inf \left\{t \geq 0: X_{t}^{\pi}<0\right\} .
$$

Note that bankruptcy may never occur under some strategies. For example, if the owners of the company inject enough capital whenever the surplus process is about to drop below 
0 to keep the surplus process at or above 0 , bankruptcy never occurs. We define $T^{\pi}=+\infty$ in this case.

Define

$$
\mathrm{P}_{x}(\cdot)=\mathrm{P}\left(\cdot \mid X_{0-}=x\right), \quad \mathrm{E}_{x}[\cdot]=\mathrm{E}\left[\cdot \mid X_{0-}=x\right] .
$$

The performance of a control strategy $\pi$ is measured by the return function defined as follows:

$$
R_{\pi}(x)=\mathrm{E}_{x}\left[\int_{0-}^{T^{\pi}} \frac{e^{-\delta t}}{1+d} \mathrm{~d} D_{t}-\int_{0-}^{T^{\pi}} \frac{e^{-\delta t}}{1-c} \mathrm{~d} C_{t}\right], x \geq 0
$$

Remark 2.2. (i) From the definition above, we can see that the class of admissible strategies, $\Pi$, includes admissible strategies under which no capital will be injected at all and strategies that inject capital before the surplus falls below 0 so that bankruptcy will never occur. For example, the strategy that prescribes to inject no capital at all and to pay out the excess of surplus over a pre-specified non-negative number as dividends is an admissible strategy. Under such strategy, the controlled surplus will fall below 0 eventually and therefore the bankruptcy time is finite. Another special admissible strategy is to distribute all the available surplus as dividends at time 0 and inject no capital at all. In this case, bankruptcy occurs immediately at time 0 , and the associated return function is $\frac{x}{1+d}$.

(ii) In our paper the shareholders of the company is not compelled to inject capital at any time, unlike in Kulenko and Schmidli (2008) where the controlled surplus is never allowed to be negative, which is guaranteed via compulsory capital injections.

For convenience, we use $X$ and $X^{\pi}$ to denote the stochastic processes $\left\{X_{t} ; t \geq 0\right\}$ and $\left\{X_{t}^{\pi} ; t \geq 0\right\}$, respectively. Note that for any admissible strategy $\pi$, the stochastic process $X^{\pi}$ is right-continuous and adapted to the filtration $\left\{\mathcal{F}_{t} ; t \geq 0\right\}$.

The objective of this paper is to study the maximal return function (also called value function):

$$
V(x)=\sup _{\pi \in \Pi} R_{\pi}(x), x \geq 0
$$

investigate the existence of optimal strategies and identify an optimal admissible strategy, if any.

\section{Auxiliary Results}

By following the standard arguments in stochastic control theory (e.g. Fleming and Soner, 1993) we can obtain the following dynamic programming principle: for any stopping time $\tau$,

$$
V(x)=\sup _{\pi \in \Pi} \mathrm{E}_{(x)}\left[\int_{0-}^{\tau} e^{-\delta t} \frac{1}{1+d} \mathrm{~d} D_{t}-\int_{0-}^{\tau} e^{-\delta t} \frac{1}{1-c} \mathrm{~d} C_{t}+e^{-\delta \tau} V\left(X_{\tau}^{\pi}\right)\right],
$$


and the associated Hamilton-Jacobi-Bellman (HJB) equation:

$$
\max \left\{\frac{\sigma^{2}(x)}{2} f^{\prime \prime}(x)+\mu(x) f^{\prime}(x)-\delta f(x), \frac{1}{1+d}-f^{\prime}(x), f^{\prime}(x)-\frac{1}{1-c}\right\}=0, x \geq 0 .
$$

Following the same lines in Section 5 of Løkka and Zervos (2008), we can conjecture that the value function may also satisfy the following boundary condition:

$$
\max \left\{-f(0), f^{\prime}(0)-\frac{1}{1-c}\right\}=0 .
$$

The HJB equation (3.6) together with the boundary equation (3.7) is closely related to the HJB equations associated with the following two auxiliary optimization problems.

\section{Auxiliary Problem I: The optimization problem without capital injections}

For the same model, now no capital injections are allowed and the objective is to maximize the expected discounted dividends until bankruptcy. This problem can be considered as finding the optimal strategy restricting to the set $\Pi_{N}$ that consists of all admissible dividend strategies in $\Pi$ with no capital injections. That is,

$$
\Pi_{N}=\left\{\left\{\left(C_{t}, D_{t}\right) ; t \geq 0\right\} \in \Pi: C_{t} \equiv 0 \text { for all } t \geq 0\right\} .
$$

Then, the value function can be defined as

$$
V_{N}(x)=\sup _{\pi \in \Pi_{N}} \mathrm{E}_{x}\left[\int_{0-}^{T^{\pi}} e^{-\delta t} \frac{1}{1+d} \mathrm{~d} D_{t}\right], x \geq 0 .
$$

Applying standard arguments in stochastic control, we can find the associated HJB:

$$
\max \left\{\frac{\sigma^{2}(x)}{2} f^{\prime \prime}(x)+\mu(x) f^{\prime}(x)-\delta f(x), \frac{1}{1+d}-f^{\prime}(x)\right\}=0, x \geq 0,
$$

Moreover, note that the value function satisfies the boundary equation:

$$
f(0)=0 .
$$

A similar optimization problem has been addressed in Shreve et al. (1984) where there is no transaction cost. More specifically, by setting $d=0$, this problem is exactly same as the "Absorption Problem" in Shreve et al. (1984) with $P=0$.

\section{Auxiliary Problem II: The Optimization Problem without Bankruptcy}

This is the situation assumes that the owners of the company are under the obligation to inject capital to prevent the company from going bankrupt. This is an optimization problem where we only consider the strategies under which the surplus process is always non-negative. In this case, bankruptcy will never occur. Define

$$
\Pi_{P}=\left\{\pi \in \Pi: X_{t}^{\pi} \geq 0 \text { for all } t \geq 0\right\} .
$$


Then, $T^{\pi}=+\infty$ for $\pi \in \Pi_{P}$. The value function, which attains the optimal value among all the strategies in $\Pi_{P}$, is defined by

$$
V_{P}(x)=\sup _{\pi \in \Pi_{P}} \mathrm{E}_{(x)}\left[\int_{0-}^{T^{\pi}} e^{-\delta t} \frac{1}{1+d} \mathrm{~d} D_{t}-\int_{0-}^{T^{\pi}} e^{-\delta t} \frac{1}{1-c} \mathrm{~d} C_{t}\right], x \geq 0 .
$$

The associated HJB equation is

$$
\max \left\{\frac{\sigma^{2}(x)}{2} f^{\prime \prime}(x)+\mu(x) f^{\prime}(x)-\delta f(x), \frac{1}{1+d}-f^{\prime}(x), f^{\prime}(x)-\frac{1}{1-c}\right\}=0, x \geq 0,
$$

and the value function satisfies the boundary equation,

$$
f^{\prime}(0)=\frac{1}{1-c} \text {. }
$$

This optimization problem corresponds to the Reflection Problem studied in Shreve et al. (1984).

We can see that the combination of all the HJB equations and the boundary equations for the two auxiliary optimization problems I and II, (3.8), (3.9), (3.10) and (3.11), is identical to the HJB equation and the boundary equation of the original optimization problem, (3.6) and (3.7). This motivates the definition and the study of the function $u_{b}$ (defined in Definition 3.2).

Definition 3.1. Define $f_{1}$ and $f_{2}$ to be the unique solutions to the following two initial value problems, respectively:

$$
\begin{aligned}
& \frac{\sigma^{2}(x)}{2} f^{\prime \prime}(x)+\mu(x) f^{\prime}(x)-\delta f(x)=0 \\
& f(0)=0, \quad f^{\prime}(0)=1
\end{aligned}
$$

and

$$
\begin{aligned}
& \frac{\sigma^{2}(x)}{2} f^{\prime \prime}(x)+\mu(x) f^{\prime}(x)-\delta f(x)=0 \\
& f(0)=1, \quad f^{\prime}(0)=1 .
\end{aligned}
$$

We can see that

$$
\begin{aligned}
& f_{1}(0)=0, f_{2}(0)=1, f_{1}^{\prime}(0)=f_{2}^{\prime}(0)=1, \\
& f_{1}^{\prime \prime}(0)=\frac{2\left(\delta f_{1}(0)-\mu(0) f_{1}^{\prime}(0)\right)}{\sigma^{2}(0)}=\frac{-2 \mu(0)}{\sigma^{2}(0)}, \\
& f_{2}^{\prime \prime}(0)=\frac{2\left(\delta f_{2}(0)-\mu(0) f_{2}^{\prime}(0)\right)}{\sigma^{2}(0)}=\frac{2(\delta-\mu(0))}{\sigma^{2}(0)} .
\end{aligned}
$$

Moreover, the functions $f_{1}(\cdot)$ and $f_{2}(\cdot)$ form a set of fundamental solutions to the second order ordinary differential equations,

$$
\frac{\sigma^{2}(x)}{2} f^{\prime \prime}(x)+\mu(x) f^{\prime}(x)-\delta f(x)=0 .
$$


Therefore, a general form of solution to (3.15) is $C_{1} f_{1}(x)+C_{2} f_{2}(x)$ where $C_{1}, C_{2}$ are two constants.

It follows by Definition 3.1 that for $i=1,2$,

$$
f_{i}^{\prime \prime}(x)=\frac{2}{\sigma^{2}(x)}\left(\delta f_{i}(x)-\mu(x) f_{i}^{\prime}(x)\right), \quad x \geq 0 .
$$

Therefore,

$$
\begin{aligned}
& f_{1}^{\prime \prime}(x) f_{2}^{\prime}(x)-f_{1}^{\prime}(x) f_{2}^{\prime \prime}(x) \\
& =\frac{2}{\sigma^{2}(x)}\left(\delta f_{1}(x)-\mu(x) f_{1}^{\prime}(x)\right) f_{2}^{\prime}(x)-\frac{2}{\sigma^{2}(x)}\left(\delta f_{2}(x)-\mu(x) f_{2}^{\prime}(x)\right) f_{1}^{\prime}(x) \\
& =\frac{2 \delta}{\sigma^{2}(x)}\left(f_{1}(x) f_{2}^{\prime}(x)-f_{2}(x) f_{1}^{\prime}(x)\right), \quad x \geq 0
\end{aligned}
$$

Define

$$
W\left(f_{1}, f_{2}\right)(x)=f_{1}(x) f_{2}^{\prime}(x)-f_{2}(x) f_{1}^{\prime}(x) .
$$

Then $W\left(f_{1}, f_{2}\right)$ is the Wronskian of the solutions $f_{1}$ and $f_{2}$. Noticing $W\left(f_{1}, f_{2}\right)(0)=$ $-1 \neq 0$ (see (3.12) ), we conclude that $W\left(f_{1}, f_{2}\right)(x)$ is always different from 0 , which along with (3.16) implies that $f_{1}^{\prime \prime}(x) f_{2}^{\prime}(x)-f_{1}^{\prime}(x) f_{2}^{\prime \prime}(x)$ is never 0 on $[0,+\infty)$.

For any fixed $b \geq 0$, use $C_{1}(b)$ and $C_{2}(b)$ to represent the solutions of $C_{1}, C_{2}$ to the following linear equations:

$$
\begin{aligned}
& C_{1} f_{1}^{\prime}(b)+C_{2} f_{2}^{\prime}(b)=\frac{1}{1+d}, \\
& C_{1} f_{1}^{\prime \prime}(b)+C_{2} f_{2}^{\prime \prime}(b)=0 .
\end{aligned}
$$

We can see that

$$
\begin{aligned}
& C_{1}(b)=-\frac{f_{2}^{\prime \prime}(b)}{(1+d)\left(f_{1}^{\prime \prime}(b) f_{2}^{\prime}(b)-f_{1}^{\prime}(b) f_{2}^{\prime \prime}(b)\right)}, \\
& C_{2}(b)=\frac{f_{1}^{\prime \prime}(b)}{(1+d)\left(f_{1}^{\prime \prime}(b) f_{2}^{\prime}(b)-f_{1}^{\prime}(b) f_{2}^{\prime \prime}(b)\right)} .
\end{aligned}
$$

As $f_{1}(\cdot)$ and $f_{2}(\cdot)$ are differentiable of infinite order. It is not hard to see that the functions $C_{1}(\cdot)$ and $C_{2}(\cdot)$ are differentiable of infinite order as well.

Definition 3.2. (i) Define the following functions:

$$
\begin{aligned}
& u_{b}(x)=C_{1}(b) f_{1}(x)+C_{2}(b) f_{2}(x), \\
& g(b)=u_{b}(0)=C_{2}(b), \\
& h(b)=u_{b}^{\prime}(0)=C_{1}(b)+C_{2}(b) .
\end{aligned}
$$

(ii) Define the quantities, $b_{N}^{*}$ and $b_{P}^{*}$, as follows:

$$
\begin{aligned}
& b_{N}^{*}= \begin{cases}\inf \left\{b \geq 0: C_{2}(b) \leq 0\right\} & \text { if } C_{2}(b) \leq 0 \text { for some } b \geq 0 \\
+\infty & \text { otherwise, }\end{cases} \\
& b_{P}^{*}= \begin{cases}\inf \left\{b \geq 0: C_{1}(b)+C_{2}(b) \geq \frac{1}{1-c}\right\} & \text { if } C_{1}(b)+C_{2}(b) \geq \frac{1}{1-c} \text { for some } b \begin{array}{l}
\text { otherwise. } \\
+\infty
\end{array}\end{cases}
\end{aligned}
$$


We can see that for any $b>0$,

$$
\begin{aligned}
& \frac{\sigma^{2}(x)}{2} u_{b}^{\prime \prime}(x)+\mu(x) u_{b}^{\prime}(x)-\delta u_{b}(x)=0, \quad 0<x<b, \\
& u_{b}^{\prime}(b)=\frac{1}{1+d}, \quad u_{b}^{\prime \prime}(b)=0 .
\end{aligned}
$$

Lemma 3.1. (i) If $0<b_{N}^{*}<+\infty$, then

$$
\begin{aligned}
& \frac{\sigma^{2}(x)}{2} u_{b_{N}^{*}}^{\prime \prime}(x)+\mu(x) u_{b_{N}^{*}}^{\prime}(x)-\delta u_{b_{N}^{*}}(x)=0,0 \leq x \leq b_{N}^{*}, \\
& u_{b_{N}^{*}}(0)=0, \quad u_{b_{N}^{*}}^{\prime}\left(b_{N}^{*}\right)=\frac{1}{1+d}, \quad u_{b_{N}^{*}}^{\prime \prime}\left(b_{N}^{*}\right)=0, \\
& u_{b_{N}^{*}}^{\prime}(x)>0, \quad u_{b_{N}^{*}}^{*}(x) \geq 0,0 \leq x \leq b_{N}^{*} .
\end{aligned}
$$

(ii) If $0<b_{P}^{*}<+\infty$, then

$$
\begin{aligned}
& \frac{\sigma^{2}(x)}{2} u_{b_{P}^{*}}^{\prime \prime}(x)+\mu(x) u_{b_{P}^{*}}^{\prime}(x)-\delta u_{b_{P}^{*}}(x)=0,0<x \leq b_{P}^{*}, \\
& u_{b_{P}^{*}}^{\prime}(0)=\frac{1}{1-c}, \quad u_{b_{P}^{*}}^{\prime}\left(b_{P}^{*}\right)=\frac{1}{1+d}, \quad u_{b_{P}^{*}}^{\prime \prime}\left(b_{P}^{*}\right)=0 .
\end{aligned}
$$

Proof. (i) Note that $C_{2}(\cdot)$ is a continuous function. It follows by (3.19) and (3.22) that

$$
u_{b_{N}^{*}}(0)=C_{2}\left(b_{N}^{*}\right)=0 \text {. }
$$

All the other equations in (3.26) and (3.27) follow immediately from (3.24) and (3.25).

Since $u_{b_{N}^{*}}(0)=0$ (see (3.31)), by Lemma 4.2 (a) in Shreve et al. (1984) (copied in Appendix), we know that $u_{b_{N}^{*}}^{\prime}$ has no zero in $\left[0, b_{N}^{*}\right]$. Therefore, by noting $u_{b_{N}^{*}}^{\prime}\left(b_{N}^{*}\right)=\frac{1}{1+d}>0$, we have $u_{b_{N}^{*}}^{\prime}(x)>0$ for $x \in\left[0, b_{N}^{*}\right]$. Then, $u_{b_{N}^{*}}(x)>u_{b_{N}^{*}}(0)=0$ for $0 \leq x \leq b_{N}^{*}$.

(ii) By using the continuity of $C_{1}(\cdot)$ and $C_{2}(\cdot)$, and the equations (3.21) and (3.23), we obtain $u_{b_{P}^{*}}^{\prime}(0)=C_{1}\left(b_{P}^{*}\right)+C_{2}\left(b_{P}^{*}\right)=\frac{1}{1-c}$. All the other equations follow immediately from (3.24) and (3.25).

Following are some properties of the functions, $f_{1}$ and $f_{2}$, which play an important role in proving the optimization result later on.

Lemma 3.2. The following properties hold:

(i) $f_{1}^{\prime}(x)>0$ for $x \geq 0$;

(ii) $f_{2}(x)>0$ and $f_{2}^{\prime}(x)>0$ for $x \geq 0$;

(iii) $f_{2}(x)>f_{1}(x)$ for $x \geq 0, f_{2}^{\prime}(x)>f_{1}^{\prime}(x)$ for $x>0$, and $f_{2}^{\prime \prime}(x)>f_{1}^{\prime \prime}(x)$ for $x \geq 0$.

Proof. (i) Note $f_{1}(0)=0$ (see (3.12)). By Lemma 4.2 (a) in Shreve et al. (1984) (copied in Appendix), we know that for any $b>0, f_{1}^{\prime}$ has no zero in $[0, b]$. Therefore, by noting $f_{1}^{\prime}(0)=1>0$ (see $(3.12)$ ), we have $f_{1}^{\prime}(x)>0$ for $x \in[0, b]$. The arbitrariness of $b$ implies $f_{1}^{\prime}(x)>0$ for $x \geq 0$. 
(ii) We first show $f_{2}(x)>0$ using proof by contradiction. Note $f_{2}(0)=1$. Suppose that there exists an $x_{0}>0$ such that $f_{2}\left(x_{0}\right) \leq 0$. Then $f_{2}$ has a zero in $\left[0, x_{0}\right]$. Then it follows by Lemma 4.2 (a) in Shreve et al. (1984) (copied in Appendix) that $f_{2}^{\prime}$ has no zero in $\left[0, x_{0}\right]$. Since $f_{2}^{\prime}(0)=1, f_{2}^{\prime}(x)>0$ for all $x \in\left[0, x_{0}\right]$. As a result, $f_{2}\left(x_{0}\right)>f_{2}(0)=1$, which contradicts the assumption that $f_{2}\left(x_{0}\right) \leq 0$.

We use proof by contradiction again to prove the positivity of $f_{2}^{\prime}$. Suppose there exists a constant $x>0$ such that $f_{2}^{\prime}(x) \leq 0$. Define $x_{1}=\inf \left\{x \geq 0: f_{2}^{\prime}(x) \leq 0\right\}$. Then by noting $f_{2}^{\prime}(0)=1$ we have $0<x_{1}<\infty, f_{2}^{\prime}\left(x_{1}\right)=0$ and

$$
f_{2}^{\prime}(x)>0 \text { for } x \in\left[0, x_{1}\right) \text {. }
$$

It follows by Lemma 4.1 in Shreve et al. (1984) (copied in Appendix) that $\left(x-x_{1}\right) f_{2}(x) f_{2}^{\prime}(x)>$ 0 for $x \in\left[0, x_{1}\right)$. Note that $f_{2}$ is always positive. We can see that $\left(x-x_{1}\right) f_{2}^{\prime}(x)>0$ for $x \in\left[0, x_{1}\right)$, which implies $f_{2}^{\prime}(x)<0$ for $x \in\left[0, x_{1}\right)$. This contradicts (3.32).

(iii) We can see that $f_{2}-f_{1}$ is a solution to $\frac{1}{2} \sigma^{2}(x) f^{\prime \prime}(x)+\mu(x) f^{\prime}(x)-\delta f(x)=0$. Then $f_{2}^{\prime}-f_{1}^{\prime}$ is a solution to the ordinary differential equation $f^{\prime \prime}(x)=\frac{2\left(\delta-\mu^{\prime}(x)\right)}{\sigma^{2}(x)} f(x)-$ $\frac{2}{\sigma^{2}(x)}\left(\mu(x)+\sigma(x) \sigma^{\prime}(x)\right) f^{\prime}(x)$. Note $f_{2}^{\prime}(0)-f_{1}^{\prime}(0)=1-1=0$. Then it follows by Lemma 4.2 (a) in Shreve et al. (1984) (copied in Appendix) that $f_{2}^{\prime \prime}-f_{1}^{\prime \prime}$ has no zero in $[0, \infty)$. Since by $(3.13)$ and (3.14) we know $f_{2}^{\prime \prime}(0)=\frac{\delta-\mu(0)}{\frac{1}{2} \sigma^{2}(0)}>\frac{-\mu(0)}{\frac{1}{2} \sigma^{2}(0)}=f_{1}^{\prime \prime}(0)$, then $f_{2}^{\prime \prime}(x)-f_{1}^{\prime \prime}(x)>0$ for all $x>0$. Hence, by noting $f_{2}^{\prime}(0)=1=f_{1}^{\prime}(0)$, it follows that $f_{2}^{\prime}(x)>f_{1}^{\prime}(x)$ for $x \geq 0$.

The inequality $f_{2}(x)>f_{1}(x)$ for $x \geq 0$ follows immediately by noting $f_{2}(0)=1>0=$ $f_{1}(0)$ and $f_{2}^{\prime}(x) \geq f_{1}^{\prime}(x)$ for $x \geq 0$.

Lemma 3.3. (i) If $\mu(0) \leq 0$, then $b_{N}^{*}=0$.

(ii) If $\mu(0)>0$, then $b_{N}^{*}>0, f_{1}^{\prime \prime}(x) \leq 0$ for $x \in\left[0, b_{N}^{*}\right)$ and $f_{1}^{\prime \prime}(x) f_{2}^{\prime}(x)-f_{1}^{\prime}(x) f_{2}^{\prime \prime}(x)<0$ for $x \in\left[0, b_{N}^{*}\right)$.

(iii) The following inequality always holds: $b_{P}^{*}>0$.

Proof. It follows by (3.18) and (3.12)-(3.14) that

$$
C_{2}(0)=\frac{f_{1}^{\prime \prime}(0)}{(1+d)\left(f_{1}^{\prime \prime}(0) f_{2}^{\prime}(0)-f_{1}^{\prime}(0) f_{2}^{\prime \prime}(0)\right)}=\frac{\frac{-\mu(0)}{\frac{1}{2} \sigma^{2}(0)}}{(1+d)\left(\frac{-\mu(0)}{\frac{1}{2} \sigma^{2}(0)}-\frac{\delta-\mu(0)}{\frac{1}{2} \sigma^{2}(0)}\right)}=\frac{\mu(0)}{\delta(1+d)}
$$

(i) Since $\mu(0) \leq 0$, it follows by (3.33) that $C_{2}(0) \leq 0$, which combined with (3.22) implies $b_{N}^{*}=0$.

(ii) Suppose $\mu(0)>0$. It follows by (3.13) and (3.33) that $f_{1}^{\prime \prime}(0)=\frac{-2 \mu(0)}{\sigma(0)}<0$ and $C_{2}(0)>0$. Hence, $b_{N}^{*}>0$ by the definition in $(3.22)$.

Now we use proof by contradiction to prove $f_{1}^{\prime \prime}(x)<0$ for $x \in\left[0, b_{N}^{*}\right)$. Suppose that there exists an $x_{0} \in\left[0, b_{N}^{*}\right)$ such that $f_{1}^{\prime \prime}\left(x_{0}\right) \geq 0$. Then we can find an $x_{1} \in\left[0, x_{0}\right]$ such 
that $f_{1}^{\prime \prime}\left(x_{1}\right)=0$, which together with $(3.18)$ implies $C_{2}\left(x_{1}\right)=0$. As a result of this and $(3.22), b_{N}^{*} \leq x_{1}$, which contradicts the fact $b_{N}^{*}>x_{0} \geq x_{1}$.

Note we just proved $f_{1}^{\prime \prime}(x)<0$ for $x \in\left[0, b_{N}^{*}\right)$ and that by the definition of $b_{N}^{*}$ in (3.22) we can see $C_{2}(x)>0$ for $x \in\left[0, b_{N}^{*}\right)$. Then it follows by $(3.18)$ that $f_{1}^{\prime \prime}(x) f_{2}^{\prime}(x)-$ $f_{1}^{\prime}(x) f_{2}^{\prime \prime}(x)<0$ for $x \in\left[0, b_{N}^{*}\right)$.

(iii) It follows by (3.17), (3.18) and (3.12)-(3.14) that

$$
C_{1}(0)+C_{2}(0)=\frac{f_{1}^{\prime \prime}(0)-f_{2}^{\prime \prime}(0)}{(1+d)\left(f_{1}^{\prime \prime}(0) f_{2}^{\prime}(0)-f_{1}^{\prime}(0) f_{2}^{\prime \prime}(0)\right)}=\frac{1}{1+d}<\frac{1}{1-c} .
$$

Then by the definition of $b_{P}^{*}$ in $(3.23)$, we can see $b_{P}^{*}>0$.

Lemma 3.4. (i) If $b_{N}^{*} \leq b_{P}^{*}<+\infty$ or $b_{N}^{*}<b_{P}^{*}=+\infty$, then $u_{b_{N}^{*}}^{\prime}(0) \leq \frac{1}{1-c}$.

(ii) If $b_{N}^{*}>b_{P}^{*}$, then $u_{b_{P}^{*}}(0) \geq 0$.

Proof. Note $b_{N}^{*} \geq 0$ and by Lemma 3.3(iii) that $b_{P}^{*}>0$.

(i) It follows by the definition of $b_{P}^{*}$ in (3.23) that

$$
C_{1}(x)+C_{2}(x) \leq \frac{1}{1-c} \text { for } x \in\left[0, b_{P}^{*}\right]-\{+\infty\} .
$$

By noticing $0 \leq b_{N}^{*} \leq b_{P}^{*}$ and $b_{N}^{*}<+\infty$ and using (3.21) and (3.34), we conclude $u_{b_{N}^{*}}^{\prime}(0)=C_{1}\left(b_{N}^{*}\right)+C_{2}\left(b_{N}^{*}\right) \leq \frac{1}{1-c}$.

(ii) It follows by $(3.20)$ and the definition of $b_{N}^{*}$ in (3.22) that

$$
u_{b}(0)=C_{2}(x)>0 \text { for } x \in\left[0, b_{N}^{*}\right)
$$

Noting that $0<b_{P}^{*}<b_{N}^{*}$, we obtain $u_{b_{P}^{*}}(0)=C_{2}\left(b_{P}^{*}\right)>0$.

Theorem 3.5. Suppose $\mu(0)>0$.

(i) If $f_{1}^{\prime \prime}(x) \geq 0$ for some $x \geq 0$, then $0<b_{N}^{*}<+\infty$ and $b_{N}^{*}=\inf \left\{x: f_{1}^{\prime \prime}(x) \geq 0\right\}$.

(ii) If $f_{1}^{\prime \prime}(x) \geq 0$ for some $x \geq 0$ and $f_{1}^{\prime}\left(b_{N}^{*}\right)<\frac{1-c}{1+d}$, then $0<b_{P}^{*}<b_{N}^{*}<+\infty$.

(iii) If $f_{1}^{\prime \prime}(x) \geq 0$ for some $x \geq 0$ and $f_{1}^{\prime}\left(b_{N}^{*}\right) \geq \frac{1-c}{1+d}$, then $b_{P}^{*} \geq b_{N}^{*}>0$ and $b_{N}^{*}<+\infty$.

(iv) If $f_{1}^{\prime \prime}(x)<0$ for all $x \geq 0$, then $b_{N}^{*}=+\infty$.

(v) If $f_{1}^{\prime \prime}(x)<0$ for all $x \geq 0$ and $\lim _{x \rightarrow \infty} f_{1}^{\prime}(x) \geq \frac{1-c}{1+d}$, then $b_{P}^{*}=+\infty$.

Proof. As $\mu(0)>0$, it follows immediately from Lemma 3.3(ii) that $b_{N}^{*}>0$.

Notice that by (3.33),

$$
C_{2}(0)=\frac{\mu(0)}{(1+d) \delta}>0
$$

(i) Since $f_{1}^{\prime \prime}(x) \geq 0$ for some $x \geq 0$, by noting that $f_{1}$ is continuously differentiable of the second order and $f_{1}^{\prime \prime}(0)=\frac{-2 \mu(0)}{(1+d) \sigma^{2}(0)}<0$ by (3.13), we can conclude that there exists an 
$x_{0}>0$ such that $f_{1}^{\prime \prime}\left(x_{0}\right)=0$. Then it follows by (3.18) that $C_{2}\left(x_{0}\right)=0$. Notice $C_{2}(0)>0$ (see (3.36)). Hence, by (3.18) and (3.22) that $0<b_{N}^{*}=\inf \left\{x \geq 0: C_{2}(x)=0\right\}=\inf \{x \geq$ $\left.0: f_{1}^{\prime \prime}(x)=0\right\}=\inf \left\{x \geq 0: f_{1}^{\prime \prime}(x) \geq 0\right\} \leq x_{0}<+\infty$.

(ii) It follows by (i) that $0<b_{N}^{*}<+\infty$ and $f_{1}^{\prime \prime}\left(b_{N}^{*}\right)=0$. Hence, it follows by (3.17) and (3.18) that $C_{1}\left(b_{N}^{*}\right)+C_{2}\left(b_{N}^{*}\right)=\frac{1}{(1+d) f_{1}^{\prime}\left(b_{N}^{*}\right)}>\frac{1}{1-c}$, where the last inequality follows by the assumption $f_{1}^{\prime}\left(b_{N}^{*}\right)<\frac{1-c}{1+d}$ and the positivity of $f_{1}^{\prime}$ (Lemma 3.2(i)). This combined with the definition of $b_{P}^{*}$ in $(3.23)$ and the continuity of $C_{1}(\cdot)+C_{2}(\cdot)$ on $(0,+\infty)$ indicates $b_{P}^{*}<b_{N}^{*}$. The strict positivity of $b_{P}^{*}$ follows immediately by Lemma 3.3(iii).

(iii) It follows immediately by (i) that $b_{N}^{*}<+\infty$. Further, it follows by (i) that

$$
f_{1}^{\prime \prime}(x)<0 \text { for } x \in\left[0, b_{N}^{*}\right), \text { and } f_{1}^{\prime \prime}\left(b_{N}^{*}\right)=0
$$

Then it follows that

$$
f_{1}^{\prime}(x)>f_{1}^{\prime}\left(b_{N}^{*}\right) \geq \frac{1-c}{1+d} \text { for } x \in\left[0, b_{N}^{*}\right) .
$$

Since $f_{2}^{\prime}(x) \geq f_{1}^{\prime}(x)$ for all $x \geq 0$ (Lemma 3.2(iii)) and $f_{1}^{\prime \prime}(x) \leq 0$ for $x \in\left[0, b_{N}^{*}\right]$ (see $(3.37))$, we obtain

$$
f_{1}^{\prime}(x) f_{2}^{\prime \prime}(x)-f_{1}^{\prime \prime}(x) f_{2}^{\prime}(x) \geq f_{1}^{\prime}(x) f_{2}^{\prime \prime}(x)-f_{1}^{\prime \prime}(x) f_{1}^{\prime}(x)>0 \text { for } x \in\left[0, b_{N}^{*}\right],
$$

where the last inequality follows by noting $f_{2}^{\prime \prime}(x)-f_{1}^{\prime \prime}(x)>0$ for $x \geq 0$ (Lemma 3.2(iii)) and $f_{1}^{\prime}(x)>0$ for $x \in\left[0, b_{N}^{*}\right]$ (see (3.38)). It follows by (3.17) and (3.18) that

$$
\begin{aligned}
C_{1}(x)+C_{2}(x) & =\frac{f_{2}^{\prime \prime}(x)-f_{1}^{\prime \prime}(x)}{(1+d)\left(f_{1}^{\prime}(x) f_{2}^{\prime \prime}(x)-f_{1}^{\prime \prime}(x) f_{2}^{\prime}(x)\right)} \\
& \leq \frac{f_{2}^{\prime \prime}(x)-f_{1}^{\prime \prime}(x)}{(1+d)\left(f_{1}^{\prime}(x) f_{2}^{\prime \prime}(x)-f_{1}^{\prime \prime}(x) f_{1}^{\prime}(x)\right)} \\
& =\frac{1}{(1+d) f_{1}^{\prime}(x)} \text { for } x \in\left[0, b_{N}^{*}\right],
\end{aligned}
$$

where the last inequality follows by noticing $f_{2}^{\prime \prime}(x)-f_{1}^{\prime \prime}(x)>0$ (Lemma 3.2(iii)) and using (3.39). Combining (3.40) and (3.38) yields

$$
C_{1}(x)+C_{2}(x)<\frac{1}{1-c} \text { for } x \in\left[0, b_{N}^{*}\right), \text { and } C_{1}\left(b_{N}^{*}\right)+C_{2}\left(b_{N}^{*}\right) \leq \frac{1}{1-c},
$$

which combined with the definition of $b_{P}^{*}$ in (3.23) implies $b_{P}^{*} \geq b_{N}^{*}$.

(iv) It follows by noticing $C_{2}(0)>0$ (see (3.36)) and the continuity of $C_{2}(\cdot)$ on $[0,+\infty)$, that either (a) $\inf \left\{b \geq 0: C_{2}(b) \leq 0\right\}=+\infty$ or (b) $0<\inf \left\{b \geq 0: C_{2}(b) \leq 0\right\}<+\infty$ and $C_{2}\left(\inf \left\{b \geq 0: C_{2}(b) \leq 0\right\}\right)=0$. That is, either (a) $b_{N}^{*}=+\infty$ or (b) $0<b_{N}^{*}<+\infty$ and $C_{2}\left(b_{N}^{*}\right)=0$. If (b) is true, then it follows by $(3.18)$ that $f_{1}^{\prime \prime}\left(b_{N}^{*}\right)=0$, which contradicts 
the assumption that $f_{1}^{\prime \prime}(x)<0$ for all $x \geq 0$. Hence (a) $b_{N}^{*}=+\infty$ is true.

(v) Notice

$$
f_{1}^{\prime}(x) f_{2}^{\prime \prime}(x)-f_{1}^{\prime \prime}(x) f_{2}^{\prime}(x) \geq f_{1}^{\prime}(x) f_{2}^{\prime \prime}(x)-f_{1}^{\prime \prime}(x) f_{1}^{\prime}(x)>0 \text { for } x \geq 0,
$$

where the first inequality follows by noting $f_{1}^{\prime \prime}(x)<0$ for all $x \geq 0$ (by assumption) and $f_{2}^{\prime}(x) \geq f_{1}^{\prime}(x)$ for all $x \geq 0$ (Lemma 3.2(iii)) and the second inequality by noting $f_{1}^{\prime}(x)>0$ and $f_{2}^{\prime \prime}(x)-f_{1}^{\prime \prime}(x)>0$ for $x \geq 0$ (see Lemma 3.2(i), (iii)). The assumption $f_{1}^{\prime \prime}(x)<0$ for all $x \geq 0$ implies that $f_{1}^{\prime}(x)$ is decreasing and

$$
f_{1}^{\prime}(x)<\lim _{x \rightarrow+\infty} f_{1}^{\prime}(x) \text { for } x \geq 0 .
$$

It follows by (3.17), (3.18), (3.42) and $f_{2}^{\prime \prime}(x)-f_{1}^{\prime \prime}(x)>0$ for $x \geq 0$ (see 3.2(iii)) that

$$
\begin{aligned}
C_{1}(x)+C_{2}(x) & =\frac{f_{2}^{\prime \prime}(x)-f_{1}^{\prime \prime}(x)}{(1+d)\left(f_{1}^{\prime}(x) f_{2}^{\prime \prime}(x)-f_{1}^{\prime \prime}(x) f_{2}^{\prime}(x)\right)} \\
& \leq \frac{f_{2}^{\prime \prime}(x)-f_{1}^{\prime \prime}(x)}{(1+d)\left(f_{1}^{\prime}(x) f_{2}^{\prime \prime}(x)-f_{1}^{\prime \prime}(x) f_{1}^{\prime}(x)\right)} \\
& =\frac{1}{(1+d)\left(f_{1}^{\prime}(x)\right)}<\frac{1}{(1+d) \lim _{x \rightarrow+\infty} f_{1}^{\prime}(x)} \\
& \leq \frac{1}{1-c}, \text { for } x \geq 0,
\end{aligned}
$$

where the second last inequality follows by noting $f_{1}^{\prime}(x)>f_{1}^{\prime}(0)>0$ (due to the assumption $f_{1}^{\prime \prime}(x)<0$ for all $x \geq 0$ and Lemma 3.2(i)) and (3.43), and the last inequality follows by the assumption $\lim _{x \rightarrow+\infty} f_{1}^{\prime}(b) \geq \frac{1-c}{1+d}$. It follows by (3.45) and the definition of $b_{P}^{*}$ in (3.23) that $b_{P}^{*}=+\infty$.

\section{The Optimality Results}

In this section, we construct two special classes of admissible strategies. We distinguish three cases: (i) when $\mu(0) \leq 0$, (ii) when $\mu(0)>0$ and $f_{1}^{\prime \prime}(x) \geq 0$ for some $x \geq 0$, and (iii) when $\mu(0)>0$ and $f_{1}^{\prime \prime}(x)<0$ for all $x>0$. We will show that in the first two cases, the optimal strategy belongs to one of the two special classes of strategies defined below, and in the last case, either there is no optimal strategy or the optimal strategy belongs to one of the two special classes. We start with defining two special types of admissible strategies.

Definition 4.1. (i) For any $b \geq 0$, define $\pi_{N}^{b}=\left\{\left(C_{t}^{N}, D_{t}^{b}\right) ; t \geq 0\right\}$ to be a strategy such that $D^{b}$ has a jump size of $\left(X_{0_{-}}-b\right)^{+}$at time 0 and then reflects the controlled surplus process at $b$, and that $C^{N} \equiv 0$.

(ii) For any $b \geq 0$, define $\pi_{P}^{b}=\left\{\left(C^{P, 0}, D^{b}\right)\right.$; $\left.t \geq 0\right\}$ to be a strategy such that $D^{b}$ has a jump size of $\left(X_{0_{-}}-b\right)^{+}$at time 0 and then reflects the controlled surplus process at $b$, and that $C^{P, 0}$ reflects the controlled surplus process at 0. 
Definition 4.2. For any $b \geq 0$, define the function $\bar{u}_{b}$ by $\bar{u}_{b}(x)=u_{b}(x)$ for $x \in[0, b]$ and $\bar{u}_{b}(x)=u_{b}(b)+\frac{x-b}{1+d}$ for $x \geq b$.

Remark 4.1. Recall that $b_{N}^{*}$ and $b_{P}^{*}$ were defined in Definition in 3.2(ii). The function $\bar{u}_{b_{N}^{*}}\left(\bar{u}_{b_{P}^{*}}\right)$ is twice continuously differentiable on $(0, \infty)$, which can be verified by noting the twice continuous differentiability of $u_{b_{N}^{*}}\left(u_{b_{P}^{*}}\right), u_{b_{N}^{*}}^{\prime}\left(b_{N}^{*}\right)=\frac{1}{1+d}\left(u_{b_{P}^{*}}^{\prime}\left(b_{P}^{*}\right)=\frac{1}{1+d}\right)$ and $u_{b_{N}^{*}}^{\prime \prime}\left(b_{N}^{*}\right)=0\left(u_{b_{P}^{*}}^{\prime \prime}\left(b_{P}^{*}\right)=0\right)$ (see (3.27) and $\left.(3.30)\right)$.

Define the operator $\mathcal{G}$ by

$$
\mathcal{G}_{f}(x)=\frac{\sigma^{2}(x)}{2} f^{\prime \prime}(x)+\mu(x) f^{\prime}(x)-\delta f(x) .
$$

Lemma 4.1. For any twice continuously differentiable function $f$ if $f$ satisfies

$$
\begin{aligned}
& f(0) \geq 0 \\
& \frac{1}{1+d} \leq f^{\prime}(x) \leq \frac{1}{1-c}, x \geq 0, \\
& \mathcal{G}_{f}(x) \leq 0, x \geq 0
\end{aligned}
$$

then $f(x) \geq V(x)$ for $x \geq 0$.

The proof of the lemma above is standard in the dividend optimization literature. First, apply Itô's Lemma to to $e^{-\delta\left(t \wedge T^{\pi}\right)} f\left(X_{t \wedge T^{\pi}}^{\pi}\right)$ for any admissible strategy and then use (4.47)(4.49) to show that $f(x)>R_{\pi}(x)$ for $x \geq 0$. The desired results follows immediately by the arbitrariness of $\pi$.

Theorem 4.2. (i) If $0<b_{N}^{*}<+\infty$, then $\bar{u}_{b_{N}^{*}}(x)=R_{\pi_{N}^{b_{N}^{*}}}(x)$ for $x \geq 0$.

(ii) If $0<b_{P}^{*}<+\infty$, then $\bar{u}_{b_{P}^{*}}(x)=R_{\pi_{P}^{b_{P}^{*}}}(x)$ for $x \geq 0$.

Proof. (i) By noticing the twice continuous differentiability of $\bar{u}_{b_{N}^{*}}(\cdot)$ and the definition for $\pi_{N}^{b_{N}^{*}}$ in Definition 4.1(i), and applying Itô's Lemma we obtain

$$
\begin{aligned}
& e^{-\delta\left(t \wedge T^{\pi_{N}^{m_{N}^{*}}}\right)} \bar{u}_{b_{N}^{*}}\left(X_{t \wedge T^{\pi_{N}^{*}}}^{\pi_{N}^{b_{N}^{*}}}\right) \\
& =\bar{u}_{b_{N}^{*}}\left(X_{0-}\right)+\int_{0-}^{t \wedge T^{\pi_{N}^{*}}} e^{-\delta s} \mathcal{G}_{\bar{u}_{b_{N}^{*}}^{*}}\left(X_{s-}^{\pi_{N}^{b_{N}^{*}}}\right) \mathrm{d} s-\int_{0-}^{t \wedge T^{\pi_{N}^{*}}} e^{-\delta s} \bar{u}_{b_{N}^{*}}^{\prime}\left(X_{s-}^{\pi_{N}^{b_{N}^{*}}}\right) \mathrm{d} D_{s}^{b_{N}^{*}} \\
& +\int_{0-}^{t \wedge T^{\pi_{N}^{*}}} e^{-\delta s} \bar{u}_{b_{N}^{*}}^{\prime}\left(X_{s-}^{\pi_{N}^{b_{N}^{*}}}\right) \mathrm{d} C_{s}^{N}+\int_{0-}^{t \wedge T^{\pi_{N}^{*}}} e^{-\delta s} \sigma\left(X_{s-}^{\pi_{N}^{b_{N}^{*}}}\right) \bar{u}_{b_{N}^{*}}^{\prime}\left(X_{s-}^{\pi_{N}^{b_{N}^{*}}}\right) \mathrm{d} W_{s} .
\end{aligned}
$$

It follows by Definition 4.1(i) for $\pi_{N}^{b_{N}^{*}}$ that

$$
\begin{aligned}
& 0 \leq X_{s-}^{\pi_{N}^{*}} \leq b_{N}^{*} \text { for } 0<s \leq T^{\pi_{N}^{n_{N}^{*}}} \\
& \mathrm{~d} D_{s}^{b_{N}^{*}}=I\left\{X_{s-}^{\pi_{N}^{b_{N}^{*}}}=b_{N}^{*}\right\} \mathrm{d} D_{s}^{b_{N}^{*}}, \quad \mathrm{~d} C_{s}^{N} \equiv 0 \text { for } 0<s \leq T^{\pi_{N}^{b_{N}^{*}}} .
\end{aligned}
$$


Noting the boundedness of $X_{s-}^{\pi_{N}^{b_{N}^{*}}} 0 \leq s \leq T^{\pi_{N}^{b_{N}^{*}}}$ (see (4.51)), we can conclude that the stochastic process $\left\{\int_{0-}^{t \wedge T^{\pi_{N}^{*}}} e^{-\delta s} \sigma\left(X_{s-}^{\pi_{N}^{b_{N}^{*}}}\right) \bar{u}_{b_{N}^{*}}^{\prime}\left(X_{s-}^{\pi_{N}^{b_{N}^{*}}}\right) \mathrm{d} W_{s} ; t \geq 0\right\}$ is a martingale and as a result, for any $t>0$,

$$
\mathrm{E}_{x}\left[\int_{0-}^{t \wedge T^{\pi_{N}^{*}}} e^{-\delta s} \sigma\left(X_{s-}^{\pi_{N}^{*}}\right) \bar{u}_{b_{N}^{*}}^{\prime}\left(X_{s-}^{\pi_{N}^{b_{N}^{*}}}\right) \mathrm{d} W_{s}\right]=0
$$

It follows by $(4.52), \bar{u}_{b_{N}^{*}}^{\prime}\left(b_{N}^{*}\right)=u_{b_{N}^{*}}^{\prime}\left(b_{N}^{*}\right)=\frac{1}{1+d}($ see $(3.27))$ and the monotone convergence that

$$
\begin{aligned}
& \lim _{t \rightarrow \infty} \mathrm{E}_{x}\left[-\int_{0-}^{t \wedge T^{\pi_{N}^{*}}} e^{-\delta s} \bar{u}_{b_{N}^{*}}^{\prime}\left(X_{s-}^{\pi_{N}^{b_{N}^{*}}}\right) \mathrm{d} D_{s}^{b_{N}^{*}}+\int_{0-}^{t \wedge T^{b_{N}^{*}}} e^{-\delta s} \bar{u}_{b_{N}^{*}}^{\prime}\left(X_{s-}^{\pi_{N}^{b_{N}^{*}}}\right) \mathrm{d} C_{s}^{N}\right] \\
& =-R_{\pi_{N}^{*}}(x) .
\end{aligned}
$$

By noting (4.51) and $\bar{u}_{b_{N}^{*}}(x)=u_{b_{N}^{*}}(x)$ for $0 \leq x \leq b_{N}^{*}$ we obtain that $\bar{u}_{b_{N}^{*}}\left(X_{s-}^{\pi_{N}^{*}}\right)=$ $u_{b_{N}^{*}}\left(X_{s-}^{\pi_{N}^{b_{N}^{*}}}\right)$ for $x \in\left[0, T^{\pi_{N}^{b_{N}^{*}}}\right]$ given $X_{0-}=x \geq 0$ and therefore

$$
\mathrm{E}_{x}\left[\int_{0-}^{t \wedge T^{\pi_{N}^{*}}} \mathcal{G}_{\bar{u}_{b_{N}^{*}}}\left(X_{s-}^{\pi_{N}^{b_{N}^{*}}}\right) \mathrm{d} s\right]=\mathrm{E}_{x}\left[\int_{0-}^{t \wedge T^{\pi_{N}^{*}}} \mathcal{G}_{u_{b_{N}^{*}}^{*}}\left(X_{s-}^{\pi_{N}^{b_{N}^{*}}}\right) \mathrm{d} s\right]=0 \text { for } x \geq 0 \text { and } s \geq 0,
$$

where the last equality follows by (3.26). Note that $\bar{u}_{b_{N}^{*}}\left(X_{T^{b_{N}^{*}}}^{\pi_{N}^{b_{N}^{*}}}\right)=\bar{u}_{b_{N}^{*}}(0)=0$ (due to (3.27)) for $x \geq 0$ and therefore,

$$
\lim _{t \rightarrow \infty} \mathrm{E}_{x}\left[e^{-\delta\left(t \wedge T^{\pi_{N}^{*}}\right)} \bar{u}_{b_{N}^{*}}\left(X^{\pi_{N}^{b_{N}^{*}}}{ }_{t \wedge T^{\pi_{N}^{*}}}^{\pi_{N}^{*}}\right)\right]=\lim _{t \rightarrow \infty} \mathrm{E}_{x}\left[e^{-\delta t} \bar{u}_{b_{N}^{*}}\left(X_{t}^{\pi_{N}^{b_{N}^{*}}}\right) I\left\{t<T^{\pi_{N}^{b_{N}^{*}}}\right\}\right]=0,
$$

where the last equality follows by noticing that $X_{t}^{\pi_{N}^{b_{N}^{*}}}, t \geq 0$, is bounded (see (4.51)) and therefore $\bar{u}_{b_{N}^{*}}\left(X_{t}^{\pi_{N}^{*}}\right), t \geq 0$, is bounded. By combining (4.50), (4.53), (4.54), (4.55) and (4.56), and noting $\mathrm{E}_{x}\left[\bar{u}_{b_{N}^{*}}\left(X_{0-}\right)\right]=\bar{u}_{b_{N}^{*}}(x)$ we derive $\bar{u}_{b_{N}^{*}}(x)=R_{\pi_{N}^{b_{N}^{*}}}(x)$ for $x \geq 0$.

(ii) By using the twice continuous differentiability of $\bar{u}_{b_{P}^{*}}$ and Itô's Lemma we obtain

$$
\begin{aligned}
e^{-\delta t} \bar{u}_{b_{P}^{*}}\left(X_{t}^{\pi_{P}^{b_{P}^{*}}}\right)= & \bar{u}_{b_{P}^{*}}\left(X_{0-}\right)+\int_{0-}^{t} e^{-\delta s} \mathcal{G}_{\bar{u}_{b_{P}^{*}}}\left(X_{s-}^{\pi_{P}^{b_{P}^{*}}}\right) \mathrm{d} s-\int_{0-}^{t} e^{-\delta s} \bar{u}_{b_{P}^{*}}^{\prime}\left(X_{s-}^{\pi_{P}^{b_{P}^{*}}}\right) \mathrm{d} D_{s}^{b_{P}^{*}} \\
& +\int_{0-}^{t} e^{-\delta s} \bar{u}_{b_{P}^{*}}^{\prime}\left(X_{s-}^{\pi_{P}^{b_{P}^{*}}}\right) \mathrm{d} C_{s}^{P, 0}+\int_{0-}^{t} e^{-\delta s} \sigma\left(X_{s-}^{\pi_{P}^{*}}\right) \bar{u}_{b_{P}^{*}}^{\prime}\left(X_{s-}^{\pi_{P}^{b_{P}^{*}}}\right) \mathrm{d} W_{s} .
\end{aligned}
$$


It follows by Definition 4.1(ii) for $\pi_{P}^{b_{P}^{*}}$ that

$$
\begin{aligned}
& T^{\pi_{P}^{*}}=+\infty \\
& 0 \leq X_{s-}^{\pi_{P}^{*}} \leq b_{P}^{*} \text { for } s>0, \\
& \mathrm{~d} D_{s}^{b_{P}^{*}}=I\left\{X_{s-}^{\pi_{P}^{*}}=b_{P}^{*}\right\} \mathrm{d} D_{s}^{b_{P}^{*}}, \quad \mathrm{~d} C_{s}^{P, 0}=I\left\{X_{s-}^{\pi_{P}^{b_{P}^{*}}}=0\right\} \mathrm{d} C_{s}^{P, 0} \text { for } s>0 .
\end{aligned}
$$

The equation (4.59) implies that $\sigma\left(X_{s-}^{\pi_{P}^{b_{P}^{*}}}\right) \bar{u}_{b_{P}^{*}}^{\prime}\left(X_{s-}^{\pi_{P}^{b_{P}^{*}}}\right), s \geq 0$, is bounded and therefore, the stochastic process $\left\{\int_{0-}^{t} e^{-\delta s} \sigma\left(X_{s-}^{\pi_{P}^{b_{P}^{*}}}\right) \bar{u}_{b_{P}^{*}}^{\prime}\left(X_{s-}^{\pi_{P}^{b_{P}^{*}}}\right) \mathrm{d} W_{s} ; t \geq 0\right\}$ is a martingale. As a result, for any $t>0$ and each $n$,

$$
\mathrm{E}_{x}\left[\int_{0-}^{t} e^{-\delta s} \sigma\left(X_{s-}^{\pi_{P}^{b_{P}^{*}}}\right) \bar{u}_{b_{P}^{*}}^{\prime}\left(X_{s-}^{\pi_{P}^{b_{P}^{*}}}\right) \mathrm{d} W_{s}\right]=0
$$

It follows by $(4.60), \bar{u}_{b_{P}^{*}}^{\prime}(0)=u_{b_{P}^{*}}^{\prime}(0)=\frac{1}{1-c}, \bar{u}_{b_{P}^{*}}^{\prime}\left(b_{P}^{*}\right)=u_{b_{P}^{*}}^{\prime}\left(b_{P}^{*}\right)=\frac{1}{1+d}($ see $(3.30))$ and the monotone convergence that

$$
\begin{aligned}
& \lim _{t \rightarrow \infty} \mathrm{E}_{x}\left[-\int_{0-}^{t} e^{-\delta s} \bar{u}_{b_{P}^{*}}^{\prime}\left(X_{s-}^{\pi_{P}^{*}}\right) \mathrm{d} D_{s}^{b_{P}^{*}}+\int_{0-}^{t} e^{-\delta s} \bar{u}_{b_{P}^{*}}^{\prime}\left(X_{s-}^{\pi_{P}^{b_{P}^{*}}}\right) \mathrm{d} C_{s}^{P, 0}\right] \\
& =\mathrm{E}_{x}\left[-\int_{0-}^{T^{\pi_{P}^{*}}} \frac{e^{-\delta s}}{1+d} \mathrm{~d} D_{s}^{b_{P}^{*}}+\int_{0-}^{T^{\pi_{P}^{*}}} \frac{e^{-\delta s}}{1-c} \mathrm{~d} C_{s}^{P, 0}\right] \\
& =-R_{\pi_{P}^{b_{P}^{*}}}(x), x \in\left[0, b_{P}^{*}\right],
\end{aligned}
$$

where the first and second last equality follows by noting $T^{\pi_{P}^{b_{P}^{*}}}=+\infty$ (see (4.58).

By noting $(4.59)$ and $\bar{u}_{b_{P}^{*}}(x)=u_{b_{P}^{*}}(x)$ for $0 \leq x \leq b_{P}^{*}$, we conclude that $\bar{u}_{b_{P}^{*}}\left(X_{s-}^{\pi_{P}^{b_{P}^{*}}}\right)=$ $u_{b_{P}^{*}}\left(X_{s-}^{\pi_{P}^{b_{P}^{*}}}\right)$ for $s>0$, given $X_{0-}=x \geq 0$, and therefore

$$
\mathrm{E}_{x}\left[\int_{0-}^{t} \mathcal{G}_{\bar{u}_{P}^{*}}\left(X_{s-}^{\pi_{P}^{*}}\right) \mathrm{d} s\right]=\mathrm{E}_{x}\left[\int_{0-}^{t} \mathcal{G}_{u_{b_{P}^{*}}}\left(X_{s-}^{\pi_{P}^{b_{P}^{*}}}\right) \mathrm{d} s\right]=0 \text { for } x \geq 0 \text { and } t \geq 0,
$$

where the last equality follows by (3.29) and (4.59). It follows by noting that $\bar{u}_{b_{P}^{*}}\left(X_{t}^{\pi_{P}^{b_{P}^{*}}}\right)$, $t \geq 0$, is bounded (due to (4.59)) that

$$
\lim _{t \rightarrow \infty} \mathrm{E}_{x}\left[e^{-\delta t} \bar{u}_{b_{P}^{*}}\left(X_{t}^{\pi_{P}^{b_{P}^{*}}}\right)\right]=0 .
$$

By combining $(4.57),(4.61),(4.62),(4.63)$ and $(4.64)$, and noting $\mathrm{E}_{x}\left[\bar{u}_{b_{P}^{*}}\left(X_{0-}\right)\right]=\bar{u}_{b_{P}^{*}}(x)$, we arrive at $\bar{u}_{b_{P}^{*}}(x)=R_{\pi_{P}^{b_{P}^{*}}}(x)$ for $x \geq \in\left[0, b_{P}^{*}\right]$. For $x>b_{P}^{*}$, the equation $\bar{u}_{b_{P}^{*}}(x)=R_{\pi_{P}^{b_{P}^{*}}}(x)$ follows immediate by the two functions involved. 


\subsection{When $\mu(0) \leq 0$}

We will show in the following that, in this situation, it is optimal to pay all the available surplus out as dividends immediately and inject no capital. Ruin occurs immediately in this case.

Theorem 4.3. If $\mu(0) \leq 0$, then $V(x)=\frac{x}{1+d}$ for $x \geq 0$ and $\pi_{N}^{0}$ is optimal.

Proof. Define $f(x)=\frac{x}{1+d}$ for $x \geq 0$. It is not hard to verify that (4.47) and (4.48) in Lemma 4.1 hold. Note that $\mathcal{G}_{f}(x)=\frac{\mu(x)}{1+d}-\frac{\delta x}{1+d} \leq \frac{\mu(0)+\delta x}{1+d}-\frac{\delta x}{1+d}=\frac{\mu(0)}{1+d} \leq 0$, where the first inequality above follows by the assumption $\mu^{\prime}(x) \leq \delta$. Then it follows by applying Lemma 4.1 that $\frac{x}{1+d}=f(x) \geq V(x)$ for $x \geq 0$. Notice that according to Definition 4.1(i), $\pi_{N}^{0}=\left(D^{0}, C^{N}\right), \mathrm{d} D_{0}^{0}=\left(X_{0-}^{\pi_{N}^{0}}\right)^{+}, \mathrm{d} C_{0}^{N}=0$ and $T^{\pi_{N}^{0}}=0$. Hence, $R_{\pi_{N}^{0}}(x)=\frac{x}{1+d}$ for $x \geq 0$. As a result, $\frac{x}{1+d} \leq \sup _{\pi \in \Pi} R_{\pi}(x)=V(x)$ for $x \geq 0$. Consequently, $V(x)=\frac{x}{1+d}$ for $x \geq 0$.

The optimality of $\pi_{N}^{0}$ follows immediately from $R_{\pi_{N}^{0}}(x)=\frac{x}{1+d}=V(x)$ for $x \geq 0$.

\subsection{When $\mu(0)>0$ and $f_{1}^{\prime \prime}(x) \geq 0$ for some $x \geq 0$}

We show that in this case, either $\pi_{N}^{b_{N}^{*}}$ is optimal, which is a strategy that prescribes to pay out the surplus in excess of $b_{N}^{*}$ as dividends and injects no capital, or $\pi_{P}^{b_{P}^{*}}$ is optimal, which is a strategy that prescribes to pay out the surplus in excess of $b_{P}^{*}$ as dividends and injects minimal amount of capital whenever the surplus is about to fall below 0 to prevent bankruptcy.

Theorem 4.4. Suppose $\mu(0)>0$. Then, $b_{N}^{*}, b_{P}^{*}>0$.

(i) If $b_{N}^{*} \leq b_{P}^{*}<+\infty$ or $b_{N}^{*}<b_{P}^{*}$, then $V(x)=\bar{u}_{b_{N}^{*}}(x)$ for $x \geq 0$ and $\pi_{N}^{b_{N}^{*}}$ is an optimal strategy.

(ii) If $b_{N}^{*}>b_{P}^{*}$, then $V(x)=\bar{u}_{b_{P}^{*}}(x)$ for $x \geq 0$ and $\pi_{P}^{b_{P}^{*}}$ is an optimal strategy.

Proof. It follows immediately by Lemma 3.3(ii) and (iii) that $b_{N}^{*}, b_{P}^{*}>0$.

(i) It follows by Theorem 4.2(i) and the fact $\pi_{N}^{b_{N}^{*}} \in \Pi$ that

$$
\bar{u}_{b_{N}^{*}}(x)=R_{\pi_{N}^{b_{N}^{*}}}(x) \leq \sup _{\pi \in \Pi} R_{\pi}(x)=V(x) \text { for } x \geq 0 .
$$

It suffices to show $\bar{u}_{b_{N}^{*}}(x) \geq V(x)$ for $x \geq 0$.

The inequality $\bar{u}_{b_{N}^{*}}(x) \geq V(x)$ for $x \geq 0$ can be proven by using the twice differentiability of $\bar{u}_{b_{N}^{*}}($ Remark 4.1), and

$$
\begin{aligned}
& \bar{u}_{b_{N}^{*}}(0) \geq 0 \text { for } x \geq 0, \\
& \frac{1}{1+d} \leq \bar{u}_{b_{N}^{*}}^{\prime}(x) \leq \frac{1}{1-c} \text { for } x \geq 0, \\
& \mathcal{G}_{\bar{u}_{b_{N}^{*}}}(x) \leq 0, \text { for } x>0
\end{aligned}
$$

and applying Lemma 4.1. 
We show in the following that

$$
\bar{u}_{b_{N}^{*}}^{\prime \prime}(x) \leq 0 \text { for } x>0
$$

The inequality above is obvious for $x \geq b_{N}^{*}$ by noting $\bar{u}_{b_{N}^{*}}^{\prime \prime}\left(b_{N}^{*}\right)=0$ (see (3.27)) and $\bar{u}_{b_{N}^{*}}(x)=\frac{x-b_{N}^{*}}{1+d}+u_{b_{N}^{*}}\left(b_{N}^{*}\right)$ for $x \geq b_{N}^{*}$. We use proof by contradiction to prove (4.68) for $x \in\left(0, b_{N}^{*}\right)$. Suppose that there exists an $x \in\left(0, b_{N}^{*}\right)$ such that $\bar{u}_{b_{N}^{*}}^{\prime \prime}(x)>0$. Therefore, there exists an $x \in\left(0, b_{N}^{*}\right)$ such that $u_{b_{N}^{*}}^{\prime \prime}(x)=\bar{u}_{b_{N}^{*}}^{\prime \prime}(x)>0$. Note by $(3.26)$ that

$$
u_{b_{N}^{*}}^{\prime \prime}(0+)=\frac{2}{\sigma^{2}(0)}\left(-\mu(0) u_{b_{N}^{*}}^{\prime}(0+)+\delta u_{b_{N}^{*}}(0)\right) \leq 0
$$

where the last inequality follows by $\mu(0)>0, u_{b_{N}^{*}}^{\prime}(0+) \geq 0$ (see $(3.28)$ ) and $u_{b_{N}^{*}}(0)=0$ (see $(3.27))$. Further note $u_{b_{N}^{*}}^{\prime \prime}\left(b_{N}^{*}\right)=0$. We can find $0 \leq x_{1}<x_{2} \leq b_{N}^{*}$ such that

$$
u_{b_{N}^{*}}^{\prime \prime}\left(x_{1}\right)=0, u_{b_{N}^{*}}^{\prime \prime}\left(x_{2}\right)=0, \quad \text { and } u_{b_{N}^{*}}^{\prime \prime}(x)>0, x \in\left(x_{1}, x_{2}\right) .
$$

It follows by (4.46), (3.26) and (4.69) that

$$
\begin{aligned}
& \mu\left(x_{1}\right) u_{b_{N}^{*}}^{\prime}\left(x_{1}\right)-\delta u_{b_{N}^{*}}\left(x_{1}\right)=\mathcal{G}_{u_{b_{N}^{*}}}\left(x_{1}\right)=0, \\
& \mu(x) u_{b_{N}^{*}}^{\prime}(x)-\delta u_{b_{N}^{*}}(x)=\mathcal{G}_{u_{b_{N}^{*}}}(x)-\frac{\sigma^{2}(x)}{2} u_{b_{N}^{*}}^{\prime \prime}(x)<0, \quad x_{1}<x<x_{2}, \\
& \mu\left(x_{2}\right) u_{b_{N}^{*}}^{\prime}\left(x_{2}\right)-\delta u_{b_{N}^{*}}\left(x_{2}\right)=\mathcal{G}_{u_{b_{N}^{*}}}\left(x_{2}\right)=0 .
\end{aligned}
$$

Therefore,

$$
\begin{aligned}
& \mu(x) u_{b_{N}^{*}}^{\prime}(x)-\delta u_{b_{N}^{*}}(x)-\left(\mu\left(x_{1}\right) u_{b_{N}^{*}}^{\prime}\left(x_{1}\right)-\delta u_{b_{N}^{*}}\left(x_{1}\right)\right)<0, x_{1}<x<x_{2}, \\
& \mu(x) u_{b_{N}^{*}}^{\prime}(x)-\delta u_{b_{N}^{*}}(x)-\left(\mu\left(x_{2}\right) u_{b_{N}^{*}}^{\prime}\left(x_{2}\right)-\delta u_{b_{N}^{*}}\left(x_{2}\right)\right)<0, \quad x_{1}<x<x_{2} .
\end{aligned}
$$

By dividing (4.70) and (4.71) by $x-x_{1}$ and $x-x_{2}$, respectively, and then letting $x \downarrow x_{1}$ and $x \uparrow x_{2}$, respectively, we arrive at

$$
\begin{aligned}
& \mu^{\prime}\left(x_{1}\right) u_{b_{N}^{*}}^{\prime}\left(x_{1}\right)+\mu\left(x_{1}\right) u_{b_{N}^{*}}^{\prime \prime}\left(x_{1}\right)-\delta u_{b_{N}^{*}}^{\prime}\left(x_{1}\right) \leq 0, \\
& \mu^{\prime}\left(x_{2}\right) u_{b_{N}^{*}}^{\prime}\left(x_{2}\right)+\mu\left(x_{2}\right) u_{b_{N}^{*}}^{\prime \prime}\left(x_{2}\right)-\delta u_{b_{N}^{*}}^{\prime}\left(x_{2}\right) \geq 0 .
\end{aligned}
$$

It follows by (4.72), (4.73) and (4.69) that

$$
\left(\delta-\mu^{\prime}\left(x_{1}\right)\right) u_{b_{N}^{*}}^{\prime}\left(x_{1}\right) \geq 0 \geq\left(\delta-\mu^{\prime}\left(x_{2}\right)\right) u_{b_{N}^{*}}^{\prime}\left(x_{2}\right) .
$$

Notice by (4.69) and (3.28) that $0<u_{b_{N}^{*}}^{\prime}\left(x_{1}\right)<u_{b_{N}^{*}}^{\prime}\left(x_{2}\right)$. As a result of this and (4.74),

$$
\delta-\mu^{\prime}\left(x_{1}\right) \geq 0 \geq \delta-\mu^{\prime}\left(x_{2}\right) .
$$

By notice that $\mu^{\prime}(x)<\delta$ for $x \geq 0$, we have $\mu^{\prime}\left(x_{1}\right)<\delta$, which is a contradiction to (4.75).

Now we proceed to show $\frac{1}{1+d} \leq \bar{u}_{b_{N}^{*}}^{\prime}(x) \leq \frac{1}{1-c}$ for $x \geq 0$. Since $\bar{u}_{b_{N}^{*}}^{\prime \prime}(x) \leq 0$ for $x>0$ $($ see $(4.68)), \bar{u}_{b_{N}^{*}}^{\prime}\left(b_{N}^{*}\right)=u_{b_{N}^{*}}^{\prime}\left(b_{N}^{*}\right)=\frac{1}{1+d}($ see $(3.27))$ and $\bar{u}_{b_{N}^{*}}^{\prime}(0)=u_{b_{N}^{*}}^{\prime}(0) \leq \frac{1}{1-c}($ by Lemma 
3.4(i)), we have $\frac{1}{1+d} \leq \bar{u}_{b_{N}^{*}}^{\prime}(x) \leq \frac{1}{1-c}$ for $x \in\left[0, b_{N}^{*}\right]$. By the definition $\bar{u}_{b_{N}^{*}}$ we can see that $\bar{u}_{b_{N}^{*}}^{\prime}(x)=\frac{1}{1+d}$ for $x \geq b_{N}^{*}$.

Next, we show that $\mathcal{G}_{\bar{u}_{b_{N}^{*}}}(x) \leq 0$ for $x \geq 0$. This inequality for $x \in\left(0, b_{N}^{*}\right)$ follows immediately by (4.46) and (3.26). Since $\bar{u}_{b_{N}^{*}}(x)=\frac{x-b_{N}^{*}}{1+d}+u_{b_{N}^{*}}\left(b_{N}^{*}\right)$ for $x \geq b_{N}^{*}$, by $(4.46)$ it follows

$$
\mathcal{G}_{\bar{u}_{b_{N}^{*}}}^{\prime}(x)=\frac{\mu^{\prime}(x)}{1+d}-\frac{\delta}{1+d} \leq 0 \text { for } x>b_{N}^{*},
$$

where the last inequality follows by the assumption $\mu^{\prime}(x)<\delta$. As a result,

$$
\mathcal{G}_{\bar{u}_{b_{N}^{*}}}(x) \leq \mathcal{G}_{\bar{u}_{b_{N}^{*}}}\left(b_{N}^{*}\right)=0 \text { for } x>b_{N}^{*},
$$

where the last equality follows by (3.26).

The optimality of $\pi_{b_{N}^{*}}$ follows by noting $V(x)=\bar{u}_{b_{N}^{*}}(x)=R_{\bar{u}_{b_{N}^{*}}}(x)$ for $x \geq 0$, where the last equality follows by Theorem 4.2(i).

(ii) It follows by Theorem 4.2(ii) and the fact $\pi_{P}^{b_{P}^{*}} \in \Pi$ that $\bar{u}_{b_{P}^{*}}(x)=R_{\pi_{P}^{b_{P}^{*}}}(x) \leq$ $\sup _{\pi \in \Pi} R_{\pi}(x)=V(x)$ for $x \geq 0$. It suffices to show $\bar{u}_{b_{P}^{*}}(x) \geq V(x)$ for $x \geq 0$. To this end, again we just need to use the twice differentiability of $\bar{u}_{b_{P}^{*}}$ (Remark 4.1), verify that

$$
\begin{aligned}
& \frac{1}{1+d} \leq \bar{u}_{b_{P}^{*}}^{\prime}(x) \leq \frac{1}{1-c} \text { for } x>0 \\
& \bar{u}_{b_{P}^{*}}(0) \geq 0 \\
& \mathcal{G}_{\bar{u}_{b_{P}^{*}}}(x) \leq 0 \text { for } x>0
\end{aligned}
$$

and then apply Lemma 4.1.

We first show

$$
\bar{u}_{b_{P}^{*}}^{\prime \prime}(x) \leq 0 \text { for } x>0
$$

The inequality abvoe for $x \geq b_{P}^{*}$ is obvious by noting $\bar{u}_{b_{P}^{*}}^{\prime \prime}\left(b_{P}^{*}\right)=0$ (see (3.30)) and $\bar{u}_{b_{P}^{*}}(x)=\frac{x-b_{P}^{*}}{1+d}+u_{b_{P}^{*}}\left(b_{P}^{*}\right)$ for $x \geq b_{P}^{*}$. We use proof by contradiction to prove (4.79) for $x \in\left(0, b_{P}^{*}\right)$. Suppose that there exists an $x \in\left(0, b_{P}^{*}\right)$ such that $\bar{u}_{b_{P}^{*}}^{\prime \prime}(x)>0$. Then, there exists an $x \in\left(0, b_{P}^{*}\right)$ such that $u_{b_{P}^{*}}^{\prime \prime}(x)=\bar{u}_{b_{P}^{*}}^{\prime \prime}(x)>0$. By noting $u_{b_{P}^{*}}^{\prime}(0)=\frac{1}{1-c}>\frac{1}{1+d}=$ $u_{b_{P}^{*}}^{\prime}\left(b_{P}^{*}\right)$ (see $\left.(3.30)\right)$ and the continuity of $\bar{u}_{b_{P}^{*}}^{\prime \prime}$, we know that $\bar{u}_{b_{P}^{*}}^{\prime \prime}$ is negative somewhere in the interval $\left[0, b_{P}^{*}\right]$. Further notice that $u_{b_{P}^{*}}^{\prime \prime}\left(b_{P}^{*}\right)=0$, therefore, we can find $x_{1}, x_{2}$ with $0 \leq x_{1}<x_{2} \leq b_{P}^{*}$ such that

$$
u_{b_{P}^{*}}^{\prime \prime}\left(x_{1}\right)=0, u_{b_{P}^{*}}^{\prime \prime}\left(x_{2}\right)=0, \quad \text { and } u_{b_{P}^{*}}^{\prime \prime}(x)>0, x \in\left(x_{1}, x_{2}\right) .
$$

By repeating the argument right below (4.69) with $b_{N}^{*}$ and $u_{b_{N}^{*}}$ there being replaced by $b_{P}^{*}$ and $u_{b_{P}^{*}}$, respectively, we can obtain the same contradiction. 
Now we proceed to show $\frac{1}{1+d} \leq \bar{u}_{b_{P}^{*}}^{\prime}(x) \leq \frac{1}{1-c}$ for $x \geq 0$. Since $\bar{u}_{b_{P}^{*}}^{\prime \prime}(x) \leq 0$ for $x>0$ $($ see $(4.79)), \bar{u}_{b_{P}^{*}}^{\prime}\left(b_{P}^{*}\right)=u_{b_{P}^{*}}^{\prime}\left(b_{P}^{*}\right)=\frac{1}{1+d}($ see $(3.30))$ and $\bar{u}_{b_{P}^{*}}^{\prime}(0)=u_{b_{P}^{*}}^{\prime}(0)=\frac{1}{1-c}($ see $(3.30))$, we have $\frac{1}{1+d} \leq \bar{u}_{b_{P}^{*}}^{\prime}(x) \leq \frac{1}{1-c}$ for $x \in\left[0, b_{P}^{*}\right]$. By the definition of $\bar{u}_{b_{P}^{*}}$ we can see that $\bar{u}_{b_{P}^{*}}^{\prime}(x)=\frac{1}{1+d}$ for $x \geq b_{P}^{*}$.

The equation $(4.77)$ ) can be proven by noting $\bar{u}_{b_{P}^{*}}(0)=u_{b_{P}^{*}}(0) \geq 0$, where the last inequality follows by Lemma 3.4 (ii).

We now proceed to show that $\mathcal{G}_{\bar{u}_{b_{P}^{*}}}(x) \leq 0$ for $x \geq 0$. This inequality for $x \in\left[0, b_{P}^{*}\right]$ follows immediately by (4.46) and (3.29). Since $\bar{u}_{b_{P}^{*}}(x)=\frac{x-b_{P}^{*}}{1+d}+u_{b_{P}^{*}}\left(b_{P}^{*}\right)$ for $x \geq b_{P}^{*}$, by (4.46) it follows that

$$
\mathcal{G}_{\bar{u}_{b_{P}^{*}}^{*}}^{\prime}(x)=\frac{\mu^{\prime}(x)-\delta}{1+d} \leq 0 \text { for } x>b_{P}^{*} .
$$

As a result, $\mathcal{G}_{\bar{u}_{b_{P}^{*}}}(x) \leq \mathcal{G}_{\bar{u}_{b_{P}^{*}}}\left(b_{P}^{*}\right)=0$ for $x>b_{P}^{*}$, where the last equality follows by (3.26).

The optimality of $\pi_{b_{P}^{*}}$ follows by noting $V(x)=\bar{u}_{b_{P}^{*}}(x)=R_{\bar{u}_{b_{P}^{*}}}(x)$ for $x \geq 0$, where the last equality follows by Theorem 4.2(ii).

The following Corollary is an immediate result of Theorem 3.5(i)-(iii) and Theorem 4.4 .

Corollary 4.5. Suppose $\mu(0)>0$.

(i) If there exists some $x \geq 0$ such that $f_{1}^{\prime \prime}(x) \geq 0$ and $f_{1}^{\prime}\left(\inf \left\{x \geq 0: f_{1}^{\prime \prime}(x) \geq 0\right\}\right)<\frac{1-c}{1+d}$, then $0<b_{P}^{*}<\infty$ and the strategy $\pi_{b_{P}^{*}}^{P}$ is optimal.

(ii) If there exists some $x \geq 0$ such that $f_{1}^{\prime \prime}(x) \geq 0$ and $f_{1}^{\prime}\left(\inf \left\{x \geq 0: f_{1}^{\prime \prime}(x) \geq 0\right\}\right) \geq \frac{1-c}{1+d}$, then $0<b_{N}^{*}=\inf \left\{x \geq 0: f_{1}^{\prime \prime}(x) \geq 0\right\}<+\infty$ and the strategy $\pi_{b_{N}^{*}}^{N}$ is optimal.

4.3. When $\mu(0)>0$ and $f_{1}^{\prime \prime}(x)<0$ for all $x>0$

In this case, $b_{N}^{*}=+\infty$ (see Theorem 3.5(iv)), and either $b_{P}^{*}<+\infty$ or $b_{P}^{*}=+\infty$. In the first case, by Theorem 4.4(ii) we know that the strategy $\pi_{P}^{b_{P}^{*}}$ is optimal. We will show that in the second case, there is no optimal strategy. To this end, we start with introducing the following new functions.

Definition 4.3. For any $b \geq 0$, define the functions $k_{b}$ and $m_{b}$ by

$$
\begin{aligned}
& k_{b}(x)= \begin{cases}\frac{f_{1}(x)}{(1+d) f_{1}^{\prime}(b)}, & 0 \leq x \leq b, \\
\frac{f_{1}(b)}{(1+d) f_{1}^{\prime}(b)}+\frac{x-b}{1+d}, & x>b,\end{cases} \\
& m_{b}(x)= \begin{cases}C_{3}(b) f_{1}(x)+C_{4}(b) f_{2}(x), & 0 \leq x \leq b, \\
C_{3}(b) f_{1}(b)+C_{4}(b) f_{2}(b)+\frac{x-b}{1+d}, & x>b,\end{cases}
\end{aligned}
$$

where $C_{3}(b)=\frac{\frac{f_{2}^{\prime}(b)}{1-c}-\frac{1}{1+d}}{f_{2}^{\prime}(b)-f_{1}^{\prime}(b)}$ and $C_{4}(b)=\frac{\frac{1}{1+d}-\frac{f_{1}^{\prime}(b)}{1-c}}{f_{2}^{\prime}(b)-f_{1}^{\prime}(b)}$.

Lemma 4.6. For any $b \geq 0, k_{b}(x)=R_{\pi_{N}^{b}}(x)$ and $m_{b}(x)=R_{\pi_{P}^{b}}(x)$ for $x \geq 0$.

Definition 4.4. Define $U_{N}^{*}$ to be the first positive solution $b$, if any, to $k_{b}^{\prime \prime}(b)=0$ and $U_{P}^{*}$ the first positive solution $b$, if any, to $m_{b}^{\prime \prime}(b)=0$. 
Remark 4.2. (i) We can see that for any $b>0$,

$$
\begin{aligned}
& \frac{\sigma^{2}(x)}{2} k_{b}^{\prime \prime}(x)+\mu(x) k_{b}^{\prime}(x)-\delta k_{b}(x)=0 \\
& k_{b}(0)=0, \quad k_{b}^{\prime}(b)=\frac{1}{1+d},
\end{aligned}
$$

and therefore, it follows by (4.4) in Shreve et al. (1984) that $k_{b}(x)$ equals $\frac{1}{1+d} V_{b}(x)$, where $V_{b}(\cdot)$ is the return function defined by (4.3) in Shreve et al. (1984) with the parameter $P$ there being set to 0 . By noting the similar structure of the strategy underlying the return function $V_{b}(\cdot)$ when setting $P=0$ and $\pi_{N}^{b}$ (see Definition 4.1), we obtain

$$
k_{b}(x)=R_{\pi_{N}^{b}}(x) \text { for } x \geq 0 .
$$

Furthermore, we can see that, $U_{N}^{*}$, if exists, coincides with the $U^{*}$ defined in Theorem 4.3 in Shreve et al. (1984), and that the case that $U_{N}^{*}$ does not exist is equivalent to the case that $U^{*}$ does not exist in the same reference. Therefore, in the case that $U_{N}^{*}$ does not exist, it follows by Theorem 4.3 in Shreve et al. (1984) that $\lim _{b \rightarrow \infty} k_{b}(x)$ exists and is finite for all $x \geq 0$. Furthermore, by the same theorem and its proof, we know that if $U_{N}^{*}$ does not exist, $k(x):=\lim _{b \rightarrow \infty} k_{b}(x)$ is twice continuously differentiable on $[0, \infty)$ and concave, and that

$$
k^{\prime}(x)>\frac{1}{1+d} \text { for } x \geq 0, \quad \mathcal{G}_{k}(x)=0 \text { for } x \geq 0 .
$$

(ii) For any $b>0$, the function $m_{b}(x)$ satisfies the following equations

$$
\begin{aligned}
& \frac{\sigma^{2}(x)}{2} m_{b}^{\prime \prime}(x)+\mu(x) m_{b}^{\prime}(x)-\delta m_{b}(x)=0 \\
& m_{b}^{\prime}(0)=\frac{1}{1-c}, \quad m_{b}^{\prime}(b)=\frac{1}{1+d},
\end{aligned}
$$

and therefore, $m_{b}(x)$ equals $\frac{1}{1+d} V_{0, b}(x)$, where $V_{0, b}(x)$ here refers to the same function defined for the reflection problem (RP) in Shreve et al. (1984) (the second last paragraph before Theorem 4.4) with the parameter $k$ there being set to $\frac{1+d}{1-c}$. By noting the similar structure of the strategy underlying the return function $V_{b}(\cdot)$ when setting $P=0$ and $\pi_{N}^{b}$ (see Definition 4.1), we obtain

$$
m_{b}(x)=R_{\pi_{P}^{b}}(x) \text { for } x \geq 0 .
$$

Furthermore, we can see that $U_{P}^{*}$, if exists, coincides with the $U^{*}$ for the $R P$ problem, which is defined in Theorem 4.5 of Shreve et al. (1984), and that the case where $U_{P}^{*}$ does not exist is equivalent to the case that $U^{*}$ does not exist.

In the case that $U_{P}^{*}$ does not exist, it follows by Theorem 4.5 in Shreve et al. (1984) that $\lim _{b \rightarrow \infty} m_{b}(x)$ exists and is finite for all $x \geq 0$. Furthermore, by the same theorem and its proof, we know that if $U_{P}^{*}$ does not exist, $m(x):=\lim _{b \rightarrow \infty} m_{b}(x)$ is twice continuously differentiable on $[0, \infty)$ and concave, and that

$$
m^{\prime}(x)>\frac{1}{1+d} \text { for } x \geq 0, \quad \mathcal{G}_{m}(x)=0 \text { for } x \geq 0 .
$$

(iii) It is not hard to see that if $b_{N}^{*}<+\infty, b_{N}^{*}=U_{N}^{*}$, and if $b_{P}^{*}<+\infty, b_{P}^{*}=U_{P}^{*}$. 
Lemma 4.7. (i) If $b_{N}^{*}=+\infty$, then $U_{N}^{*}$ does not exist. (ii) If $b_{P}^{*}=+\infty$, then $U_{P}^{*}$ does not exist.

Proof. (i) We use proof by contradiction. Suppose $U_{N}^{*}$ exists. Then according to the definition of $U_{N}^{*}$ in Definition 4.4, we know that

$$
0 \leq U_{N}^{*} \leq+\infty, \quad k_{U_{N}^{*}}^{\prime \prime}\left(U_{N}^{*}\right)=0 .
$$

Notice by the definition of $k_{b}$ in Definition 4.3 that

$$
\begin{aligned}
& \frac{\sigma^{2}(x)}{2} k_{U_{N}^{*}}^{\prime \prime}(x)+\mu(x) k_{U_{N}^{*}}^{\prime}(x)-\delta k_{U_{N}^{*}}(x)=0,0<x<U_{N}^{*}, \\
& k_{U_{N}^{*}}(0)=0, \quad k_{U_{N}^{*}}^{\prime}\left(U_{N}^{*}\right)=\frac{1}{1+d} .
\end{aligned}
$$

Then by the definition of $b_{N}^{*}$ in Definition 3.2 and (4.88)-(4.90) we conclude $b_{N}^{*} \leq U_{N}^{*}<$ $+\infty$, which contradicts the assumption $b_{N}^{*}=+\infty$.

(ii) We apply similar argument. Suppose $U_{P}^{*}$ exists. Then according to the definition of $U_{P}^{*}$ in Definition 4.4, we know that

$$
0 \leq U_{P}^{*}<+\infty, m_{U_{P}^{*}}^{\prime \prime}\left(U_{P}^{*}\right)=0 .
$$

Notice by the definition of $m_{b}$ in Definition 4.3 that

$$
\begin{aligned}
& \frac{\sigma^{2}(x)}{2} m_{U_{P}^{*}}^{\prime \prime}(x)+\mu(x) m_{U_{P}^{*}}^{\prime}(x)-\delta m_{U_{P}^{*}}(x)=0,0<x<U_{P}^{*}, \\
& m_{U_{P}^{*}}^{\prime}(0)=\frac{1}{1-c}, \quad m_{U_{P}^{*}}^{\prime}\left(U_{P}^{*}\right)=\frac{1}{1+d},
\end{aligned}
$$

which combined with (4.91) and the definition of $b_{P}^{*}$ in Definition 4.4 imply $b_{P}^{*} \leq U_{P}^{*}<$ $+\infty$. This is an contradiction to the assumption $b_{P}^{*}=+\infty$.

Theorem 4.8. Suppose $\mu(0)>0$ and $f_{1}^{\prime \prime}(x)<0$ for all $x>0$. Then, $b_{N}^{*}=+\infty$. (i) If $\lim _{x \rightarrow+\infty} f_{1}^{\prime}(x) \leq \frac{1-c}{1+d}$, then either (a) $0<b_{P}^{*}<+\infty$ or (b) $b_{P}^{*}=+\infty$. In case (a), $V(x)=\bar{u}_{b_{P}^{*}}(x)$, and in case (b) $V(x)=\lim _{b \rightarrow+\infty} m_{b}(x)$ for $x \geq 0$ and there is no optimal strategy.

(ii) If $\lim _{x \rightarrow+\infty} f_{1}^{\prime}(x) \geq \frac{1-c}{1+d}$, then $V(x)=\lim _{b \rightarrow+\infty} k_{b}(x)$ for $x \geq 0$ and there is no optimal strategy.

Proof. It follows by Theorem 3.5 (iv) that $b_{N}^{*}=+\infty$.

(i) (a) The result follows immediately by noticing $b_{P}^{*}<+\infty=b_{N}^{*}$ and using Theorem 4.4(ii).

(b) It follows by Lemma 4.7 that $U_{P}^{*}$ does not exist and therefore by 4.2 (ii) that $m(x):=$ $\lim _{b \rightarrow+\infty} m_{b}(x)$ exists and is finite for all $x \geq 0$. Notice that $m_{b}(x)=R_{\pi_{P}^{b}}(x) \leq$ $\sup _{\pi \in \Pi} R_{\pi}(x)=V(x)$, where the first equality follows by (4.86). As a result, $m(x) \leq V(x)$ 
for $x \geq 0$. Hence, it suffices to show that $m(x) \geq V(x)$ for $x \geq 0$. By Remark 4.2(ii) we can see that $m(x)$ is twice continuously differentiable and concave, and

$$
m^{\prime}(x)>\frac{1}{1+d} \text { for } x \geq 0, \quad \mathcal{G}_{m}(x)=0 \text { for } x \geq 0 .
$$

It follows by (4.81) and noting $f_{1}(0)=0$ and $f_{2}(0)=1$ (see (3.12)) that

$$
m(0)=\lim _{b \rightarrow+\infty} m_{b}(0)=\lim _{b \rightarrow+\infty} \frac{\frac{1}{1+d}-\frac{f_{1}^{\prime}(b)}{1-c}}{f_{2}^{\prime}(b)-f_{1}^{\prime}(b)} \geq 0,
$$

where the last inequality follows by using the assumption $\lim _{x \rightarrow+\infty} f_{1}^{\prime}(x) \leq \frac{1-c}{1+d}$ and noting $f_{2}^{\prime}>f_{1}^{\prime}$ (see Lemma 3.2(iii)). For any $x>0$ and $0<h<x$, it follows by the concavity of $m$ on $[0, \infty)$ that

$$
m^{\prime}(x) \leq \frac{m(x)-m(x-h)}{h}=\lim _{b \rightarrow+\infty} \frac{m_{b}(x)-m_{b}(x-h)}{h} \leq \lim _{b \rightarrow \infty} m_{b}^{\prime}(0)=\frac{1}{1-c},
$$

where the last equality follows by (4.85). Therefore, we can apply Lemma 4.1, which yields $m(x) \geq V(x)$ for $x \geq 0$. Consequently, $m(x)=V(x)$ for $x \geq 0$.

We now proceed to show that there is no optimal strategy in $\Pi$. We use proof by contradiction. Suppose there exists an optimal admissible strategy, denoted by $\hat{\pi}=$ $(\hat{D}, \hat{C})$. Then,

$$
V(x)=\mathrm{E}_{x}\left[\int_{0-}^{T^{\hat{\pi}}} \frac{e^{-\delta t}}{1+d} \mathrm{~d} \hat{D}_{t}-\int_{0-}^{T^{\hat{\pi}}} \frac{e^{-\delta t}}{1-c} \mathrm{~d} \hat{C}_{t}\right] .
$$

Notice that $V(x)=m(x)$ is twice continuously differentiable. The stochastic process

$$
\left\{\int_{0-}^{t} e^{-\delta s} \sigma\left(X_{s-}^{\hat{\pi}}\right) V^{\prime}\left(X_{s-}^{\hat{\pi}}\right) \mathrm{d} W_{s} ; t \geq 0\right\}
$$

is a local martingale and therefore we can find a positive sequence $t_{n} \uparrow+\infty$ such that for each $n$ the process $\left\{\int_{0-}^{t \wedge t_{n}} e^{-\delta s} \sigma\left(X_{s-}^{\hat{\pi}}\right) V^{\prime}\left(X_{s-}^{\hat{\pi}}\right) \mathrm{d} W_{s} ; t \geq 0\right\}$ is a martingale. By the optional sampling theorem we can obtain that for any $t>0$ and each $n$,

$$
\mathrm{E}_{x}\left[\int_{0-}^{t \wedge t_{n} \wedge T^{\hat{\pi}}} e^{-\delta s} \sigma\left(X_{s-}^{\hat{\pi}}\right) V^{\prime}\left(X_{s-}^{\hat{\pi}}\right) \mathrm{d} W_{s}\right]=0 .
$$

Note that $V(x) \geq \frac{x}{1+d}$ (see Remark 2.2) and therefore $\left\{\hat{D}_{t} ; t \geq 0\right\}$ is not identically 0 . Applying Itô's Lemma to $e^{-\delta\left(t \wedge t_{n} \wedge T^{\hat{\pi}}\right)} V\left(X_{t \wedge \wedge t_{n} \wedge T^{\hat{\pi}}}\right)$ leads to

$$
\begin{aligned}
& e^{-\delta\left(t \wedge t_{n} \wedge T^{\hat{\pi}}\right)} V\left(X_{t \wedge t_{n} \wedge T^{\hat{\pi}}}^{\hat{\pi}}\right) \\
= & V\left(X_{0-}\right)+\int_{0-}^{t \wedge t_{n} \wedge T^{\hat{\pi}}} e^{-\delta s} \mathcal{G}_{V}\left(X_{s-}^{\hat{\pi}}\right) \mathrm{d} s-\int_{0-}^{t \wedge t_{n} \wedge T^{\hat{\pi}}} e^{-\delta s} V^{\prime}\left(X_{s-}^{\hat{\pi}}\right) \mathrm{d} \hat{D}_{s} \\
& +\int_{0-}^{t \wedge t_{n} \wedge T^{\hat{\pi}}} e^{-\delta s} V^{\prime}\left(X_{s-}^{\hat{\pi}}\right) \mathrm{d} \hat{C}_{s}+\int_{0-}^{t \wedge t_{n} \wedge T^{\hat{\pi}}} e^{-\delta s} \sigma\left(X_{s-}^{\hat{\pi}}\right) V^{\prime}\left(X_{s-}^{\hat{\pi}}\right) \mathrm{d} W_{s} .
\end{aligned}
$$


By taking expectation on both sides of (4.97) and then using (4.96), $V(x) \geq \frac{x}{1+d} \geq 0$ (see Remark 2.2) and $\mathcal{G}_{V}(x)=\mathcal{G}_{m}(x)=0$ for $x \geq 0$ (due to (4.92)) we obtain

$$
V(x) \geq \mathrm{E}_{x}\left[\int_{0-}^{t \wedge t_{n} \wedge T^{\hat{\pi}}} e^{-\delta s} V^{\prime}\left(X_{s-}^{\hat{\pi}}\right) \mathrm{d} \hat{D}_{s}-\int_{0-}^{t \wedge t_{n} \wedge T^{\hat{\pi}}} e^{-\delta s} V^{\prime}\left(X_{s-}^{\hat{\pi}}\right) \mathrm{d} \hat{C}_{s}\right] .
$$

Notice $\mathrm{d} \hat{D}_{s} \geq 0$ and $\mathrm{d} \hat{C}_{s} \geq 0$. Letting $t \rightarrow+\infty$ and using the monotone convergence for each integral term inside the expectation, we have

$$
V(x) \geq \mathrm{E}_{x}\left[\int_{0-}^{T^{\hat{\pi}}} e^{-\delta s} V^{\prime}\left(X_{s-}^{\hat{\pi}}\right) \mathrm{d} \hat{D}_{s}-\int_{0-}^{T^{\hat{\pi}}} e^{-\delta s} V^{\prime}\left(X_{s-}^{\hat{\pi}}\right) \mathrm{d} \hat{C}_{s}\right] .
$$

Noticing that $V^{\prime}(x)=m^{\prime}(x) \in\left(\frac{1}{1+d}, \frac{1}{1+c}\right]$ (see (4.92) and (4.94)), that $\left\{\hat{D}_{t} ; t \geq 0\right\}$ is not identically 0 , we arrive at

$$
V(x)>\mathrm{E}_{x}\left[\int_{0-}^{T^{\hat{\pi}}} \frac{e^{-\delta s}}{1+d} \mathrm{~d} \hat{D}_{s}-\int_{0-}^{T^{\hat{\pi}}} \frac{e^{-\delta s}}{1-c} \mathrm{~d} \hat{C}_{s}\right]
$$

which is a contradiction to (4.95).

(ii) Noting $b_{N}^{*}=+\infty$, it follows by Lemma 4.7 that $U_{N}^{*}=+\infty$. By Remark 4.2(i) we can see that $k(x):=\lim _{b \rightarrow+\infty} k_{b}(x)$ exists, is finite, twice continuously differentiable and concave on $[0, \infty)$, and satisfies

$$
k^{\prime}(x)>\frac{1}{1+d} \text { for } x \geq 0, \quad \mathcal{G}_{k}(x)=0 \text { for } x \geq 0 .
$$

Since $f_{1}^{\prime \prime}(x)<0$ for $x \geq 0$,

$$
f_{1}^{\prime}(b)>\lim _{x \rightarrow+\infty} f_{1}^{\prime}(x) \geq \frac{1-c}{1+d}
$$

It follows by (4.80), (3.12) and (4.102) that

$$
k_{b}^{\prime}(0)=\frac{f_{1}^{\prime}(0)}{(1+d) f_{1}^{\prime}(b)}=\frac{1}{(1+d) f_{1}^{\prime}(b)} \leq \frac{1}{1-c}, b>0 .
$$

For any $x>0$ and $0<h<x$, it follows by the concavity of $k$ on $[0, \infty)$ that

$$
k^{\prime}(x) \leq \frac{k(x)-k(x-h)}{h}=\lim _{b \rightarrow+\infty} \frac{k_{b}(x)-k_{b}(x-h)}{h} \leq \lim _{b \rightarrow \infty} k_{b}^{\prime}(0) \leq \frac{1}{1-c} .
$$

As $k$ is twice continuously differentiable, we obtain

$$
k^{\prime}(0+)=\lim _{x \downarrow 0} k^{\prime}(x) \leq \frac{1}{1-c} \text { for } x \geq 0 .
$$

Further, it follows by (4.92) that

$$
k(0)=\lim _{b \rightarrow+\infty} k_{b}(0)=0
$$


which combined with (4.101), (4.103) and (4.104) implies that we can apply Lemma 4.1 for the function $k(x)$, which yields $k(x) \geq V(x)$ for $x \geq 0$. As a result, $V(x)=k(x)$ for $x \geq 0$.

We can show that there is no optimal strategy following the same lines in the paragraph containing (4.95) in (i) with $m(\cdot)$ there being replaced with $k(\cdot)$.

\section{Examples}

Example 1. (Brownian motion) Let $\mu(x)=\mu$ and $\sigma(x)=\sigma$ with $\mu>0$ and $\sigma>0$.

Let $\theta_{1}$ and $-\theta_{2}$ denote respectively the positive and negative roots to the equation $\frac{\sigma^{2}}{2} x^{2}+\mu x-\delta=0$. Then solutions of $\frac{\sigma^{2}}{2} f^{\prime \prime}(x)+\mu f^{\prime}(x)-\delta=0$ have the following general form

$$
f(x)=C_{1} e^{\theta_{1} x}+C_{2} e^{-\theta_{2} x} .
$$

Noting $f_{1}(0)=0$ and $f_{1}^{\prime}(0)=1$, we obtain

$$
f_{1}(x)=\frac{e^{\theta_{1} x}-e^{-\theta_{2} x}}{\theta_{1}+\theta_{2}}, x \geq 0 .
$$

It follows by noting $\frac{1}{\theta_{1}+\theta_{2}} \log \frac{\theta_{2}^{2}}{\theta_{1}^{2}}>0, f_{1}\left(\frac{1}{\theta_{1}+\theta_{2}} \log \frac{\theta_{2}^{2}}{\theta_{1}^{2}}\right)=0$ and $f_{1}(x)<0$ for $x \in\left[0, \frac{1}{\theta_{1}+\theta_{2}} \log \frac{\theta_{2}^{2}}{\theta_{1}^{2}}\right)$, and applying Theorem 3.5(i) that

$$
b_{N}^{*}=\frac{1}{\theta_{1}+\theta_{2}} \log \frac{\theta_{2}^{2}}{\theta_{1}^{2}}
$$

(i) Suppose $f_{1}^{\prime}\left(b_{N}^{*}\right) \geq \frac{1-c}{1+d}$, i.e.,

$$
\frac{1}{\theta_{1}+\theta_{2}}\left(\theta_{1}\left(\frac{\theta_{1}}{\theta_{2}}\right)^{-\frac{2 \theta_{1}}{\theta_{1}+\theta_{2}}}+\theta_{2}\left(\frac{\theta_{1}}{\theta_{2}}\right)^{-\frac{2 \theta_{2}}{\theta_{1}+\theta_{2}}}\right) \geq \frac{1-c}{1+d} .
$$

It follows by Theorem 3.5(iii) and Corollary 4.5(ii) that $0<b_{N}^{*} \leq b_{P}^{*}$ and therefore, the strategy $\pi_{N}^{b_{N}^{*}}$ with $b_{N}^{*}=\frac{1}{\theta_{1}+\theta_{2}} \log \frac{\theta_{2}^{2}}{\theta_{1}^{2}}$ is optimal and $V(x)=R_{\pi_{N}^{b_{N}^{*}}}(x)=k_{b_{N}^{*}}(x)$ for $x \geq 0$ (see Lemma 4.6). By Remark 4.2(i)\&(iii) and Definition 4.4 we can see that $V(x)=k_{b_{N}^{*}}(x)$ is the solution to the following equations

$$
\begin{aligned}
& \frac{\sigma^{2}}{2} f^{\prime \prime}(x)+\mu f^{\prime}(x)-\delta=0,0<x \leq b_{N}^{*}, \\
& f(0)=\frac{1}{1-c}, f^{\prime}\left(b_{N}^{*}\right)=\frac{1}{1+d} .
\end{aligned}
$$

Therefore,

$$
V(x)= \begin{cases}\frac{f_{1}(x)}{f_{1}^{\prime}\left(b_{N}^{*}\right)(1+d)}=\frac{e^{\theta_{1} x}-e^{-\theta_{2} x}}{\theta_{1}\left(\frac{\theta_{2}}{\theta_{1}}\right)^{\frac{2 \theta_{1}}{\theta_{1}+\theta_{2}}}+\theta_{2}\left(\frac{\theta_{1}}{\theta_{2}}\right)^{\frac{2 \theta_{2}}{\theta_{1}+\theta_{2}}}}, & 0 \leq x \leq b_{N}^{*} \\ V\left(b_{N}^{*}\right)+\frac{x-b_{N}^{*}}{1+d} & x>b_{N}^{*} .\end{cases}
$$


(ii) Suppose $f_{1}^{\prime}\left(b_{N}^{*}\right)<\frac{1-c}{1+d}$, i.e.,

$$
\frac{1}{\theta_{1}+\theta_{2}}\left(\theta_{1}\left(\frac{\theta_{1}}{\theta_{2}}\right)^{-\frac{2 \theta_{1}}{\theta_{1}+\theta_{2}}}+\theta_{2}\left(\frac{\theta_{1}}{\theta_{2}}\right)^{-\frac{2 \theta_{2}}{\theta_{1}+\theta_{2}}}\right)<\frac{1-c}{1+d} .
$$

It follows by Theorem 3.5(ii) and Corollary 4.5(i) that $0<b_{P}^{*}<+\infty$ and therefore the strategy $\pi_{P}^{b_{P}^{*}}$ is optimal and $V(x)=R_{\pi_{P}^{b_{P}^{*}}}(x)=m_{b_{P}^{*}}(x)$ for $x \geq 0$ (see Lemma 4.6). By Remark $4.2\left(\right.$ ii) $\&\left(\right.$ iii) and Definition 4.4 we can see that $\left(b_{P}^{*}=U_{P}^{*}, V(x)=m_{b_{P}^{*}}(x)\right)$ is the solution to the following equations

$$
\begin{aligned}
& \frac{\sigma^{2}}{2} f^{\prime \prime}(x)+\mu f^{\prime}(x)-\delta=0,0<x \leq b, \\
& f^{\prime}(0)=\frac{1}{1-c}, f^{\prime}(b)=\frac{1}{1+d}, f^{\prime \prime}(b)=0, \\
& f(x)=f(b)+\frac{x}{1+d}, x \geq b .
\end{aligned}
$$

Therefore,

$$
V(x)= \begin{cases}C_{3} e^{\theta_{1} x}+C_{4} e^{-\theta_{2} x}, & 0 \leq x \leq b_{P}^{*} \\ C_{3} e^{\theta_{1} b_{P}^{*}}+C_{4} e^{-\theta_{2} b_{P}^{*}}+\frac{x-b_{P}^{*}}{1+d} & x>b_{P}^{*},\end{cases}
$$

where $b_{P}^{*}$ is the first positive solution to $\frac{(1+d)\left(1+\theta_{1}\right) \theta_{2} e^{\theta_{1} x}}{(1-c)\left(\theta_{2}+\theta_{1} e^{\left(\theta_{1}+\theta_{2}\right) x}\right)}=1$,

$$
C_{3}=-\frac{\theta_{2}}{(1-c) \theta_{1}\left(\theta_{2}+\theta_{1} e^{\left.\left(\theta_{1}+\theta_{2}\right) b_{P}^{*}\right)}\right.}, \quad C_{4}=-\frac{\theta_{1}^{2}}{\theta_{2}^{2}} e^{\left(\theta_{1}+\theta_{2}\right) b_{P}^{*}} C_{3} .
$$

Example 2. (Geometric Brownian motion) Let $\mu(x)=a_{1} x+a_{2}$ and $\sigma(x)=\theta\left(a_{1} x+a_{2}\right)$ where $a_{1}, a_{2}$ and $\theta$ are constants with $0<a_{1}<\delta, a_{2}>0$.

Define $r_{1}$ and $r_{2}$ to be the respective positive and negative roots of $\frac{1}{2} \theta^{2} a_{1}^{2} r^{2}+r\left(a_{1}-\right.$ $\left.\frac{1}{2} \theta^{2} a_{1}^{2}\right)-\delta=0$. We can show that

$$
\begin{aligned}
& r_{1}=\frac{\frac{1}{2} \theta^{2} a_{1}-1+\sqrt{\left(1-\frac{1}{2} \theta^{2} a_{1}\right)^{2}+2 \delta \theta^{2}}}{\theta^{2} a_{1}}>1, \\
& r_{1}>0>r_{2}, \\
& r_{2}\left(r_{2}-1\right)>r_{1}\left(r_{1}-1\right) .
\end{aligned}
$$

As show in Shreve et al. (1984), solutions of

$$
\frac{\sigma^{2}(x)}{2} f^{\prime \prime}(x)+\mu(x) f^{\prime}(x)-\delta f(x)=0
$$

have a general form $f(x)=c_{1}\left(a_{1} x+a_{2}\right)^{r_{1}}+c_{2}\left(a_{1} x+a_{2}\right)^{r_{2}}, x \geq 0$. Noticing from Remark $4.2(\mathrm{i}) \&\left(\right.$ iii) and Definition 4.4 that if $b_{N}^{*} \in(0,+\infty)$, then $b_{N}^{*}=U_{N}^{*}, V(x)=k_{b_{N}^{*}}(x)$, and the pair $\left(u_{b_{N}^{*}}(\cdot), b_{N}^{*}\right)$ is the solution of $(f(\cdot), b)$ with $b$ being the smallest positive number 
among all the solutions, to (5.112) with boundary conditions $f(0)=0, f^{\prime}(b)=\frac{1}{1+d}$ and $f^{\prime \prime}(b)=0$. After some algebraic calculations we obtain

$$
\begin{aligned}
& b_{N}^{*}=\frac{a_{2}}{a_{1}}\left(\left(\frac{r_{2}\left(r_{2}-1\right)}{r_{1}\left(r_{1}-1\right)}\right)^{\frac{1}{r_{1}-r_{2}}}-1\right)<+\infty, \\
& k_{b_{N}^{*}}(x)= \begin{cases}c_{1}\left(a_{1} x+a_{2}\right)^{r_{1}}+c_{2}\left(a_{1} x+a_{2}\right)^{r_{2}} & 0 \leq x \leq b_{N}^{*}, \\
c_{1}\left(a_{1} b_{N}^{*}+a_{2}\right)^{r_{1}}+c_{2}\left(a_{1} b_{N}^{*}+a_{2}\right)^{r_{2}}+\frac{x-b_{N}^{*}}{1+d} & x>b_{N}^{*},\end{cases}
\end{aligned}
$$

where

$$
\begin{aligned}
c_{1} & =\frac{1}{(1+d) a_{1}\left(r_{1}\left(a_{1} b_{N}^{*}+a_{2}\right)^{r_{1}-1}-r_{2} a_{2}^{r_{1}-r_{2}}\left(a_{1} b_{N}^{*}+a_{2}\right)^{r_{2}-1}\right)}, \\
c_{2} & =-a_{2}^{r_{1}-r_{2}} c_{1} .
\end{aligned}
$$

We can also see from $4.2(\mathrm{i}) \&(\mathrm{iii})$ and Definition 4.4 that if $b_{P}^{*}<+\infty$, the pair $\left(u_{b_{P}^{*}}(\cdot), b_{P}^{*}\right)$ is the solution of $(f(\cdot), b)$ with $b$ being the smallest positive number to all the solutions, to (5.112) with boundary conditions $f^{\prime}(0)=\frac{1}{1-c}, f^{\prime}(b)=\frac{1}{1+d}$ and $f^{\prime \prime}(b)=0$. Therefore, $b_{P}^{*}$, if finite, is the smallest positive solution of

$$
(1+d)\left(r_{2}-r_{1}\right)\left(a_{1} x+a_{2}\right)^{r_{1}-1}+(1-c) \frac{r_{1}-1}{r_{2}-1} a_{2}^{r_{2}-1}\left(a_{1} x+a_{2}\right)^{r_{1}-r_{2}}-(1-c) a_{2}^{r_{1}-1}=0 .
$$

By noting the positivity of all parameters except for $r_{2}$ in the equation above, $1-c>0$, $r_{1}>1$ and $r_{1}>0>r_{2}$, we can show that the left-hand side of the equation is negative for $x \geq 0$ and therefore $b_{P}^{*}=+\infty$. Since $b_{N}^{*}<b_{P}^{*}$, we conclude that the strategy $\pi_{N}^{b_{N}^{*}}$ is optimal and

$$
V(x)=\bar{u}_{b_{N}^{*}}(x)= \begin{cases}c_{1}\left(a_{1} x+a_{2}\right)^{r_{1}}+c_{2}\left(a_{1} x+a_{2}\right)^{r_{2}} & 0 \leq x \leq b_{N}^{*}, \\ c_{1}\left(a_{1} b_{N}^{*}+a_{2}\right)^{r_{1}}+c_{2}\left(a_{1} b_{N}^{*}+a_{2}\right)^{r_{2}}+\frac{x-b_{N}^{*}}{1+d} & x>b_{N}^{*},\end{cases}
$$

We can see that in this case, it is not optimal to inject capital at all at any stage.

\section{Concluding Remarks}

In this study we have considered the optimal control of capital injection and dividend distribution for a class of diffusion models in presence of bankruptcy risk, where the drift and diffusion coefficients are general functions of the level of the surplus. The drift and diffusion coefficients are Lipschitz continuous, differentiable and grow at most linearly on $[0, \infty)$, and the derivative of the drift coefficient is bounded by the force of interest $\delta$. This is an extension of the studies that consider the exact same problem for simpler models (for example, Brownian motion model) or the same problem for the diffusion model (simpler or not) subject to the constraint of without bankruptcy (by assuming that the company injects capital whenever the bankruptcy is going to occur). We have distinguished different cases and discussed when there will be optimal strategies and when not. We have 
shown that in the cases where there exists an optimal strategy:

(a) the dividend payment component of the optimal strategy is of a barrier form,

(b) the optimal capital injection strategy is either

(i) to always inject capital when and only when the surplus process is about to drop below 0 without this capital injections at an amount that is just enough to prevent the surplus process from entering $(-\infty, 0)$ immediately, or

(ii) to never inject any capital at all.

In the case where there is no optimal strategy, the value function is the limiting function of the return function associated with the strategy of similar forms with the barrier converging to $+\infty$.

\section{APPENDIX}

Lemma A.1(Lemma 4.1 in Shreve et al. (1984)) Let $k$ be a solution, not identically zero, to the equation $k^{\prime \prime}(x)=\gamma(x) k(x)+\delta(x) k^{\prime}(x)$ on some interval $[a, b]$. Assume $\gamma(\cdot)$ is Lipschitz continuous and nonnegative. If, for some $\bar{x} \in[a, b], k(\bar{x})>0$ and $k^{\prime}(\bar{x}) \leq 0$, then $k^{\prime}(x) \leq 0$ for $a \leq x \leq \bar{x}$. If $k$ has a zero in $[a, b]$, then $k^{\prime}$ has no zero in $[a, b]$. If $\gamma(x)>0$ for all $x$ and for some $\bar{x}, k^{\prime}(\bar{x})=0$, then $(x-\bar{x}) k(x) k^{\prime}(x)>0$ for $x \in[a, b]$, $x \neq \bar{x}$.

Lemma A.2 (Lemma 4.2 (a) in Shreve et al. (1984)) Let $f$ be a solution, not identically constant, to the equation $\beta f(x)=\alpha(x) f^{\prime}(x)+\frac{1}{2} \sigma^{2}(x) f^{\prime \prime}(x)$ on some interval $[a, b]$. Assume $\alpha^{\prime}(\cdot) \leq \beta$. If $f$ has a zero in $[a, b]$, then $f^{\prime}$ has no zero in $[a, b]$.

Acknowledgements. We thank the anonymous referees and the Editor for their insightful comments and suggestions. We acknowledge with thanks the support from the Hong Kong Special Administrative Region (project No. HKU 705313P), Society of Actuaries' Centers of Actuarial Excellence Research Grant and the Australian Business School Special Research Grants.

Albrecher, H. and Thonhauser, S. (2009). Optimality results for dividend problems in insurance. Rev. R. Acad. Cien. Serie A. Mat., 103:295-320.

Alvarez, L. H. and Virtanen, J. (2006). A class of solvable stochastic dividend optimization problems: on the general impact of flexibility on valuation. Economic Theory, 28:373398.

Asmussen, S., Høgaard, B., and Taksar, M. (2000). Optimal risk control and dividend distribution policies. example of excess-of loss reinsurance for an insurance corporation. Finance and Stochastics, 4:299-324. 
Avanzi, B. (2009). Strategies for dividend distributions: a review. North American Actuarial Journal, 13:217-251.

Bäuerle, N. (2004). Approximation of optimal reinsurance and dividend payout policies. Mathematical Finance, 14(1):99-113.

Cadenillas, A., Choulli, T., Taksar, M. I., and Zhang, L. (2006). Classical and impulse stochastic control for the optimization of the dividend and risk policies of an insurance firm. Mathematical Finance, 16:181-202.

Cadenillas, A., Sarkar, S., and Zapatero, F. (2007). Optimal dividend policy with meanreverting cash reservoir. Mathematical Finance, 17:81-109.

Fleming, W. H. and Soner, H. M. (1993). Controlled Markov processes and viscosity solutions. Applications of Mathematics. Springer-Verlag, New York.

Guo, X., Liu, J., and Zhou, X. Y. (2004). A constrained non-linear regular-singular stochastic control problem, with applications. Stochastic Processes and their Applications, 109(2):167 - 187 .

He, L. and Liang, Z. (2008). Optimal financing and dividend control of the insurance company with proportional reinsurance policy. Insurance: Mathematics and Economics, $42(3): 976-983$.

He, L. and Liang, Z. (2009). Optimal financing and dividend control of the insurance company with fixed and proportional transaction costs. Insurance: Mathematics and Economics, 44(1):88 - 94 .

Højgaard, B. and Taksar, M. (2001). Optimal risk control for a large corporation in the presence of returns on investments. Finance and Stochastics, 5(4):527-547.

Kulenko, N. and Schmidli, H. (2008). Optimal dividend strategies in a Cramér-Lundberg model with capital injections. Insurance: Mathematics and Economics, 43:270-278.

Løkka, A. and Zervos, M. (2008). Optimal dividend and issuance of equity policies in the presence of proportional costs. Insurance: Mathematics and Economics, 42:954-961.

Meng, H. and Siu, T. K. (2011). Optimal mixed impulse-equity insurance control problem with reinsurance. SIAM Journal on Control and Optimization, 49(1):254-279.

Paulsen, J. (2008). Optimal dividend payments and reinvestments of diffusion processes with both fixed and proportional costs. SIAM Journal on Control and Optimization, 47(5):2201-2226.

Yang, R. , Liu, K. , and Xia B. (2005). Optimal impulse and regular control strategies for proportional reinsurance problem. Journal of Applied Mathematics and Computing, 18(1-2):145-158. 
Schmidli, H. (2008). Stochastic control in insurance. Springer-Verlag, London.

Sethi, S. P. and Taksar, M. I. (2002). Optimal financing of a corporation subject to random returns. Mathematical Finance, 12(2):155-172.

Shreve, S. E., Lehoczky, J. P., and Gaver, D. P. (1984). Optimal consumption for general diffusions with absorbing and reflecting barriers. SIAM Journal on Control and Optimization, 22(1):55-75.

Zhu, J. (2015). Dividend optimization for general diffusions with restricted dividend payment rates. Scandinavian Actuarial Journal, 2015 (7):592-615. 